\title{
FITOSSOCIOLOGIA DAS ESPÉCIES ARBÓREAS NATIVAS DE CERRADO EM ÁREAS ADJACENTES A DEPÓSITOS DE RESÍDUOS DOMICILIARES
}

\author{
Otacílio Antunes Santana ${ }^{1}$, José Imaña-Encinas ${ }^{2}$ \\ ${ }^{1}$ Biólogo, Dr., Depto. Geografia, UnB, Brasília, DF, Brasil - otaciliosantana@ gmail.com \\ ${ }^{2}$ Eng. Florestal, Ph.D., Depto. Engenharia Florestal, UnB, Brasília, DF, Brasil - imana@unb.br \\ Recebido para publicação: 02/07/2008 - Aceito para publicação: 25/05/2009
}

\begin{abstract}
Resumo
Os depósitos de resíduos domiciliares são construídos em áreas adjacentes a fitofisionomias nativas. Os objetivos deste trabalho foram, em áreas adjacentes a aterros e em áreas-controle, realizar e comparar o levantamento da composição das espécies arbóreas nativas de Cerrado. A presença de aterros sanitários não influenciou na diversidade arbórea nativa de Cerrado, mas na distribuição espacial das espécies. Três padrões para distribuição dos valores de importância, a partir do aterro para regiões mais distantes, foram observados: i) aumento dos valores; ii) redução dos valores; e iii) indiferente, ou seja, os valores não aumentaram e nem reduziram.

Palavras-chave: Lixo; Cerrado stricto sensu; biodiversidade; fitossociologia.
\end{abstract}

\begin{abstract}
Phytosociology of the Cerrado tree species in nearby sanitary landfill areas. The sanitary landfills are established in the adjacent areas of the native phytophysiognomies. This work aimed in adjacent areas of the sanitary landfills, and in control areas to sample and to compare the composition of Cerrado tree species. The landfill presence did not influence in the native tree species of Cerrado, and its presence influenced the spatial distribution of the species. Three standards to value importance were observed, from landfill to farther areas: i) enhance of the values; ii) reduction of the values; and iii) not significant enhance or reduction.

Keywords: Waste; Cerrado stricto sensu; biodiversity; phytosociology.
\end{abstract}

\section{INTRODUÇÃO}

A deposição de nutrientes, particularmente de nitrogênio e fósforo, em áreas de Cerrado, provenientes de áreas de aterro adjacentes, causam evidentes mudanças na abundância relativa de grupos funcionais de plantas, influenciando na biomassa e na regeneração de indivíduos arbóreos e em todo o processo de distribuição espacial das espécies (BUSTAMANTE et al., 2006; NARDOTO et al., 2006; CORADIN et al., 2002).

O Cerrado apresenta a maior riqueza florística, entre as savanas mundiais (KLINK; MOREIRA, 2002), sendo reconhecido internacionalmente como área prioritária para a conservação da biodiversidade do planeta (MITTERMEIER, et al., 1999), por abrigar mais de 6.000 espécies vegetais em suas variadas fitofisionomias (MENDONÇA et al., 1998). No Cerrado stricto sensu, ocorrem aproximadamente 230 a 250 espécies de plantas vasculares por hectare (SILBERBAUER-GOTTSBERGER; EITEN, 1983). A distribuição das espécies no Cerrado ocorre em mosaicos, com poucas espécies dominantes (cerca de vinte) e as demais raras (LÍBANO; FELFILI, 2006), sendo que espécies abundantes em uma área podem ser raras ou ausentes em outras (RATTER; DARGIE, 1992).

Estudos florísticos, fitossociológicos e fitogeográficos têm sido importantes para avaliar a diversidade e a similaridade entre as áreas de Cerrado stricto sensu, abordando variações em escala espacial, como foi demonstrado nos trabalhos de Neri et al. (2007), Balduíno et al. (2005), Teixeira et al. (2004), Assunção; Felfili (2004), Fonseca; Silva Jr. (2004), Saporetti Jr. et al. (2003), Andrade et al. (2002) e Felfili et al. (2002). A densidade da vegetação foi indicada como o fator mais importante para a 
diferenciação entre áreas de Cerrado stricto sensu, sendo, portanto, um fator importante a ser considerado para a tomada de decisão em relação às estratégias de proteção das populações e comunidades vegetais.

Os objetivos deste trabalho foram: i) realizar o levantamento da composição das espécies arbóreas nativas de Cerrado; ii) calcular os parâmetros fitossociológicos e índices de diversidade e de similaridade; e iii) fazer uma análise comparativa entre as áreas de estudo.

\section{MATERIAIS E MÉTODOS}

Os pontos amostrais foram escolhidos por estarem próximos aos depósitos de resíduos domiciliares (aterros), cobertos com vegetação nativa: um no Parque Nacional de Brasília, distante $15 \mathrm{~m}$

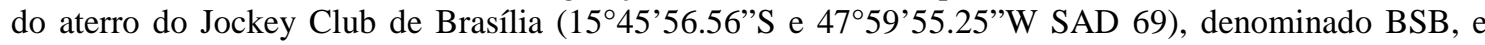
outro localizado na Chácara São Joaquim, também a 15 m do aterro sanitário de Goiânia (16³9’09.77”S e 49 23'37.08”'W SAD 69), denominado GYN. Para a amostragem-controle, outras duas áreas foram estabelecidas: uma no próprio Parque Nacional de Brasília, distante $15 \mathrm{~km}$ do aterro (BSB - Controle: 15³8'11.45”S e 48 01'55.61'W SAD 69), e outra em Goiânia, na área do Campus II da Universidade Católica de Goiás (GYN - Controle: 16³7'48.29”'S e 49¹2'46.48”W SAD 69), a cerca de 15 km do aterro.

O aterro do Jockey Club de Brasília foi criado e implementado há 35 anos, e atualmente recebe diariamente cerca de 1.800 toneladas de resíduos domiciliares (SEDUH, 2007). O aterro sanitário de Goiânia entrou em funcionamento em 1983, e atualmente recebe por dia cerca de 1.200 toneladas de resíduos domiciliares (PREFEITURA DE GOIÂNIA, 2007).

As áreas escolhidas ficam na região Centro-Oeste, com clima Aw em Brasília e Cwa em Goiânia, de acordo com a classificação de Köppen. A pluviosidade e temperatura anual média são de $1.440 \mathrm{~mm}$ e $22{ }^{\circ} \mathrm{C}$, respectivamente, para as áreas estabelecidas. Duas estações são definidas para essas tipologias climáticas: uma úmida e chuvosa, de outubro a março, e outra seca, de abril a setembro.

As altitudes das áreas de amostragem foram: BSB 797 m; BSB - Controle 838 m; GYN 1.115 m; GYN - Controle $1.150 \mathrm{~m}$. As declividades das parcelas nas áreas amostradas não ultrapassam a 15\%, correspondentes a um relevo suave ondulado.

Os solos nas regiões estudadas, tanto em Goiânia como em Brasília, foram classificados de Latossolos Vermelho-Escuros, segundo o Sistema Brasileiro de Classificação dos Solos (EMBRAPA, 1999; SANTOS et al., 2003).

A análise granulométrica das duas áreas estabelecidas classificou o solo como argiloso, com valores aproximados de $380 \mathrm{~g} \cdot \mathrm{kg}^{-1}$ de argila, $182 \mathrm{~g} \cdot \mathrm{kg}^{-1}$ de silte e $438 \mathrm{~g} \cdot \mathrm{kg}^{-1}$ de areia, com diâmetro médio ponderado de $2,81 \mathrm{~mm}$, apresentando alta taxa de infiltração hídrica, de cerca de $90 \mathrm{~cm} \cdot \mathrm{h}^{-1}$ (SANTANA e IMAÑA-ENCINAS, 2004).

As quatro áreas amostradas estão cobertas com vegetação típica de Cerrado stricto sensu, conforme classificação fitofisionômica de Eiten (2001).

Os critérios para escolha das áreas-controle foram i) o mesmo tipo de solo e ii) a densidade arbórea da vegetação nativa, em áreas próximas aos depósitos de lixo.

Nos aterros estudados e nas áreas-controle foram estabelecidas três parcelas de 25 x $500 \mathrm{~m}$ de comprimento, que foram divididas em dez subparcelas de $25 \times 50 \mathrm{~m}$, das quais foram coletados os dados de vegetação (Figura 1). O espaçamento entre as parcelas foi de $75 \mathrm{~m}$. Cada subparcela foi denominada com um número, e o mesmo procedimento foi realizado para a área controle, na qual a parte inicial das parcelas foi determinada de forma aleatória.

O levantamento da composição das espécies nativas foi realizado para todos os indivíduos arbóreos com diâmetro maior que $5 \mathrm{~cm}$, a $30 \mathrm{~cm}$ do solo (CHAPMAN, 1977), localizados nas parcelas estabelecidas em áreas adjacentes de cada aterro e nas áreas determinadas para o controle (Figura 1). As espécies arbóreas nativas foram identificadas, seus diâmetros foram mensurados com fita métrica (circunferência) e a altura medida com hipsômetro de Haga. A identificação foi feita pelo método de comparação de exsicatas (BREYER; TSUBOI, 1996) nos Herbários da Universidade de Brasília (UB) e da Reserva Ecológica do Instituto Brasileiro de Geografia e Estatística (IBGE). Quando não se obteve a identificação, o material foi enviado a um especialista em taxonomia. Os indivíduos arbóreos foram identificados por espécie, gênero e família, pelo sistema APG II (Angiosperm Phylogeny Group II, 2003). 


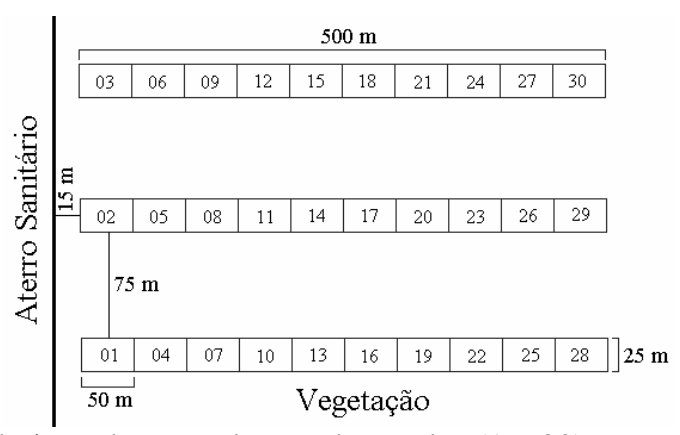

Figura 1. Esquema de distribuição das parcelas e subparcelas (1 a 30) para coleta de dados próximos aos aterros e áreas-controle. Adaptado de Carter (1993) e Marguran (1988).

Figure 1. Scheme of plots and subplots distribution (from 1 to 30) to collect the datas, nearby landfill and control areas. Adapted of Carter (1993) and of Marguran (1988).

Os parâmetros fitossociológicos, densidade relativa (DR), frequência relativa (FR), dominância relativa (DoR) e valor de importância (VI) foram calculados segundo Mueller-Dombois e Ellemberg (2002). Para a avaliação do número de espécies das comunidades, foram utilizados os índices de diversidade de Simpson e de Shannon (MARGURRAN, 1988), calculados pelo programa MSVP versão 2.1 (KOVACH, 1993).

Utilizou-se o índice de similaridade para a análise do nível de fusão (similaridade), de Sørensen, que considera a presença e a ausência das espécies, e o índice de Morisita para identificar o número de indivíduos por espécie nas subparcelas (MARGURRAN, 1988). Os resultados dos índices variaram de zero a um, em que um significa duas parcelas totalmente similares e zero significa que não há espécies em comum entre as parcelas comparadas.

Foi elaborada a classificação por UPGMA (Unweighted Pair Group Method with Arithmetic Mean), a fim de se obter um dendrograma, baseado na média aritmética dos índices de similaridade, visando às hierarquias entre os grupos formados. Em geral, os índices de similaridade maiores do que 0,5 são considerados altos (KENT; COKER, 1992). O programa FITOPAC (SHEPHERD, 1987) foi utilizado para as análises de similaridade.

A curva espécie-área pelas subparcelas amostradas foi realizada a partir da primeira subparcela, considerando-se o número de espécies encontrado. A esse valor foram acrescidas novas espécies de cada subparcela amostrada sequencialmente. Foi utilizada a equação $\mathrm{y}=\beta_{0}+\beta_{1} \cdot \operatorname{Ln}(\mathrm{x})+\varepsilon$ para verificação da suficiência amostral (COLWELL e CODDINGTON, 1994), calculando-se os coeficientes $\left(\beta_{0}\right.$ e $\left.\beta_{1}\right)$ e os parâmetros da equação $\left(\mathrm{R}^{2}=\right.$ coeficiente de determinação, $\varepsilon=$ erro do ajuste e $\mathrm{p}=$ nível de significância). Valores significativos $\left(\mathrm{R}^{2}>0,5 ; \varepsilon<0,25\right.$ e $\left.\mathrm{p}<0,05\right)$ representam a suficiência amostral.

$\mathrm{O}$ ajuste do número de indivíduos arbóreos presentes nas parcelas por centro de classe de diâmetro e os parâmetros da equação foram calculados pela equação de Meyer (1952): $Y_{j}=e^{\beta_{0}+\beta_{1} \cdot D_{j}}+\mathcal{E}$, sendo $Y_{j}$ o estimador do número de árvores por hectare na j-ésima classe diamétrica, $\beta_{0}$ e $\beta_{l}$ os coeficientes da equação, $D_{j}$ o diâmetro correspondente ao centro da j-ésima classe diamétrica e $e$ a constante dos logaritmos neperianos. Os ajustes foram calculados pelo programa Statistica 5.0.

O teste estatístico não-paramétrico Kruskal-Wallis foi utilizado para comparação dos índices de diversidade entre as subparcelas, em cada área, com nível de significância de 5\% (ZAR, 1999).

\section{RESULTADOS E DISCUSSÃO}

A densidade dos indivíduos arbóreos por hectare encontrados nas áreas estudadas foi de 475 e 556 ind/ha, para áreas mais próximas e mais distantes do aterro do Jockey Club de Brasília, respectivamente, e 449 e 505 ind/ha para áreas mais próximas do aterro sanitário de Goiânia e para áreacontrole em Goiânia, respectivamente.

O número de espécies encontradas nas áreas de estudo dos aterros foi de 70 em Brasília e $73 \mathrm{em}$ Goiânia (Tabelas 1 e 3), bem próximos aos números encontrados nas respectivas áreas-controle, de 71 e 61 (Tabelas 2 e 4), e presentes na literatura em levantamentos fitossociológicos (Tabela 5), na mesma fitofisionomia estudada e com metodologias de coleta de dados individual da vegetação semelhantes à utilizada neste trabalho. 
Tabela 1. Espécies, famílias e parâmetros fitossociológicos das árvores amostradas no Parque Nacional de Brasília, área próxima do aterro.

Table 1. Species, families and phytossociologic parameters of the sampled trees at the National Park of Brasília City, nearby landfill area.

\begin{tabular}{|c|c|c|c|c|c|c|}
\hline Espécie & Família & $\mathbf{N}^{\circ}$ ind. & DR & FR & DoR & VI \\
\hline Miconia albicans (Sw.) Triana & MELASTOMATACEAE & 198 & 10,00 & 11,56 & 10,59 & 32,15 \\
\hline Stryphnodendron adstringens (Mart.) Coville & FABACEAE & 126 & 6,50 & 6,00 & 11,55 & 24,05 \\
\hline Guapira noxia (Netto) Lundell & NYCTAGINACEAE & 90 & 4,50 & 5,08 & 12,09 & 21,67 \\
\hline Kielmeyera coriacea (Spreng.) Mart. & CLUSIACEAE & 123 & 6,83 & 6,09 & 4,08 & 17,00 \\
\hline Enterolobium contortisiliquum (Vell.) Morong & MIMOSACEAE & 105 & 5,83 & 5,07 & 6,69 & 16,59 \\
\hline Byrsonima crassa Nied. & MALPIGHIACEAE & 87 & 4,68 & 4,70 & 5,12 & 14,50 \\
\hline Qualea grandiflora Mart. & VOCHYSIACEAE & 96 & 5,33 & 4,09 & 3,59 & 13,01 \\
\hline Byrsonima verbascifolia (L.) DC. & MALPIGHIACEAE & 66 & 3,62 & 3,57 & 5,00 & 12,19 \\
\hline Miconia ferruginata DC. & MELASTOMATACEAE & 93 & 5,16 & 4,00 & 1,96 & 11,12 \\
\hline Styrax ferrugineus Nees \& Mart. & STYRACACEAE & 62 & 3,50 & 3,76 & 2,83 & 10,09 \\
\hline Anacardium occidentale $\mathrm{L}$. & ANACARDIACEAE & 60 & 3,33 & 3,20 & 2,92 & 9,45 \\
\hline Sclerolobium paniculatum Vog. var. subvelutinum Benth & CAESALPINIACEAE & 57 & 3,16 & 3,38 & 1,75 & 8,29 \\
\hline Qualea multiflora Mart. & VOCHYSIACEAE & 57 & 3,16 & 3,38 & 1,72 & 8,26 \\
\hline Aspidosperma macrocarpon Mart. & APOCYNACEAE & 48 & 2,66 & 2,63 & 2,52 & 7,81 \\
\hline Caryocar brasiliense Camb. & CARYOCARACEAE & 51 & 2,83 & 3,01 & 0,90 & 6,74 \\
\hline Vochysia thyrsoidea Pohl & VOCHYSIACEAE & 51 & 2,83 & 2,44 & 1,35 & 6,62 \\
\hline Kielmeyera neriifolia Camb. & CLUSIACEAE & 18 & 1,00 & 1,12 & 4,08 & 6,20 \\
\hline Hymenaea stigonocarpa Mart. ex Hayne & CAESALPINIACEAE & 45 & 2,50 & 2,25 & 0,70 & 5,45 \\
\hline Solanum lycocarpum A. St.-Hil. & SOLANACEAE & 30 & 1,66 & 1,88 & 1,16 & 4,70 \\
\hline Pouteria torta (Mart.) Radlk. & SAPOTACEAE & 24 & 1,33 & 1,50 & 1,61 & 4,44 \\
\hline Erythroxylum suberosum A. St.-Hil. & ERYTHROXYLACEAE & 12 & 0,66 & 0,37 & 3,12 & 4,15 \\
\hline Rapanea coriacea $\mathrm{R}$. Br. ex Roem. \& Scult. & MYRSINACEAE & 21 & 1,16 & 1,31 & 0,41 & 2,88 \\
\hline Machaerium acutifolium Vog. & FABACEAE & 21 & 1,16 & 1,31 & 0,36 & 2,83 \\
\hline Dipteryx alata Vog. & FABACEAE & 18 & 1,00 & 1,12 & 0,71 & 2,83 \\
\hline Acosmium dasycarpum (Vog.) Yakov. & FABACEAE & 18 & 1,00 & 1,12 & 0,69 & 2,81 \\
\hline Roupala montana Aubl. & PROTEACEAE & 21 & 1,16 & 1,31 & 0,33 & 2,80 \\
\hline Xylopia aromatica (Lam.) Mart. & ANNONACEAE & 9 & 0,50 & 0,56 & 1,38 & 2,44 \\
\hline Campomanesia xanthocarpa Berg. & MYRTACEAE & 12 & 0,66 & 0,75 & 1,02 & 2,43 \\
\hline Strychnos pseudoquina A. St.-Hil. & LOGANIACEAE & 15 & 0,83 & 0,75 & 0,19 & 1,77 \\
\hline Diospyros hispida DC. & EBENACEAE & 15 & 0,83 & 0,75 & 0,17 & 1,75 \\
\hline Aspidosperma verbascifolium M. Arg. & APOCYNACEAE & 9 & 0,50 & 0,56 & 0,60 & 1,66 \\
\hline Aegiphila lhotzkyana Cham. & VERBENACEAE & 12 & 0,66 & 0,75 & 0,23 & 1,64 \\
\hline Bauhinia mollis (Bong.) Walp. & CAESALPINIACEAE & 6 & 0,33 & 0,37 & 0,90 & 1,60 \\
\hline Mimosa laticifera Rizz. e Mattos Filho & MIMOSACEAE & 9 & 0,50 & 0,56 & 0,50 & 1,56 \\
\hline Aspidosperma tomentosum Mart. & APOCYNACEAE & 8 & 0,50 & 0,56 & 0,44 & 1,50 \\
\hline Zeyheria montana Mart. & BIGNONIACEAE & 7 & 0,50 & 0,56 & 0,22 & 1,28 \\
\hline Protium heptaphyllum (Aubl.) March & BURSERACEAE & 9 & 0,50 & 0,56 & 0,15 & 1,21 \\
\hline Cabralea cangerana (Vell.) Mart. & MELIACEAE & 6 & 0,33 & 0,37 & 0,50 & 1,20 \\
\hline Dalbergia miscolobium Benth. & FABACEAE & 5 & 0,33 & 0,37 & 0,43 & 1,13 \\
\hline Salvertia convallarieodora A. St.-Hil. & VOCHYSIACEAE & 5 & 0,33 & 0,37 & 0,26 & 0,96 \\
\hline Hanconia speciosa Gomez & APOCYNACEAE & 5 & 0,33 & 0,37 & 0,19 & 0,89 \\
\hline Palicourea rigida Kunth. & RUBIACEAE & 6 & 0,33 & 0,37 & 0,17 & 0,87 \\
\hline Rudgea viburnoides (Cham.) Benth. & RUBIACEAE & 6 & 0,33 & 0,37 & 0,17 & 0,87 \\
\hline Solanum crinitum Lam. & SOLANACEAE & 6 & 0,33 & 0,37 & 0,08 & 0,78 \\
\hline Symplocos lanceolata (Mart.) A. DC. & SYMPLOCACEAE & 3 & 0,16 & 0,18 & 0,42 & 0,76 \\
\hline Callisthene major Mart. & VOCHYSIACEAE & 6 & 0,33 & 0,37 & 0,06 & 0,76 \\
\hline Anacardium humile A. St.-Hil. & ANACARDIACEAE & 3 & 0,16 & 0,18 & 0,37 & 0,71 \\
\hline Didymopanax macrocarpum (Cham. \& Schlecht.) Seem. & ARALIACEAE & 1 & 0,16 & 0,18 & 0,30 & 0,64 \\
\hline Pseudobombax longiflorum (Mart. \& Zucc.) A. Robyns & BOMBACACEAE & 1 & 0,16 & 0,18 & 0,17 & 0,51 \\
\hline Dimorphandra gardneriana Tul. & CAESALPINIACEAE & 1 & 0,16 & 0,18 & 0,17 & 0,51 \\
\hline Eriotheca gracilipes (K. Schum.) A. Robyns & MALVACEAE & 1 & 0,16 & 0,18 & 0,17 & 0,51 \\
\hline Rhodocalyx rotundifolius M. Arg. & APOCYNACEAE & 1 & 0,16 & 0,18 & 0,17 & 0,51 \\
\hline Tabebuia roseo-alba (Ridley) Sandwith & BIGNONIACEAE & 1 & 0,16 & 0,18 & 0,17 & 0,51 \\
\hline Vernonia ferruginea Less. & ASTERACEAE & 1 & 0,16 & 0,18 & 0,17 & 0,51 \\
\hline Inga cf. affinis DC. & MIMOSACEAE & 1 & 0,16 & 0,18 & 0,17 & 0,51 \\
\hline Annona coriacea Mart. & ANNONACEAE & 1 & 0,16 & 0,18 & 0,17 & 0,51 \\
\hline
\end{tabular}




\begin{tabular}{lllllll}
\hline Eriotheca pubescens (Mart. \& Zucc.) Schott \& Endl. & MALVACEAE & 1 & 0,16 & 0,18 & 0,17 & 0,51 \\
Pisonia ambigua Heimerl. & NYCTAGINACEAE & 1 & 0,16 & 0,18 & 0,17 & 0,51 \\
Bowdichia virgilioides H.B.K. & FABACEAE & 1 & 0,16 & 0,18 & 0,17 & 0,51 \\
Tocoyena formosa K. Schum. & RUBIACEAE & 1 & 0,16 & 0,18 & 0,17 & 0,51 \\
Cissampelos ovalifolia DC. & MENISPERMACEAE & 1 & 0,16 & 0,18 & 0,17 & 0,51 \\
Psidium pohlianus Camb. & MYRTACEAE & 1 & 0,16 & 0,18 & 0,17 & 0,51 \\
Anadenanthera colubrina (Vell.) Brenan & MIMOSACEAE & 1 & 0,16 & 0,18 & 0,17 & 0,51 \\
Cayaponia tayuya (Vell.) Cogn. & CUCURBITACEAE & 1 & 0,16 & 0,18 & 0,17 & 0,51 \\
Macrosiphonia longiflora (Desf.) M. Arg. & APOCYNACEAE & 1 & 0,16 & 0,18 & 0,17 & 0,51 \\
Não identificadas & & 4 & 1,30 & 1,46 & 0,53 & 3,29 \\
\hline
\end{tabular}

Tabela 2. Espécies, famílias e parâmetros fitossociológicos das árvores amostradas no Parque Nacional de Brasília, área-controle.

Table 2. Species, families and phytossociologic parameters of the sampled trees at the National Park of Brasília City, control area.

\begin{tabular}{|c|c|c|c|c|c|c|}
\hline Espécie & Família & $\mathbf{N}^{\circ}$ ind & DR & FR & DoR & VI \\
\hline Alibertia macrophylla K. Schum. & RUBIACEAE & 189 & 9,00 & 8,19 & 10,24 & 27,43 \\
\hline Rudgea viburnoides (Cham.) Benth. & RUBIACEAE & 137 & 6,50 & 6,59 & 10,21 & 23,30 \\
\hline Piptocarpha rotundifolia (Less.) Baker & ASTERACEAE & 119 & 5,66 & 5,52 & 7,70 & 18,88 \\
\hline Qualea grandiflora Mart. & VOCHYSIACEAE & 119 & 5,66 & 5,52 & 7,08 & 18,26 \\
\hline Dalbergia miscolobium Benth. & FABACEAE & 137 & 6,50 & 5,75 & 3,46 & 15,71 \\
\hline Miconia albicans (Sw.) Triana & MELASTOMATACEAE & 119 & 5,16 & 5,34 & 4,37 & 14,87 \\
\hline Byrsonima intermedia A. Juss. & MALPIGHIACEAE & 109 & 5,16 & 5,16 & 3,94 & 14,26 \\
\hline Palicourea rigida Kunth. & RUBIACEAE & 105 & 4,50 & 3,81 & 3,24 & 11,55 \\
\hline Ouratea spectabilis (Mart. ex Engl.) Engl. & OCHNACEAE & 63 & 3,00 & 2,67 & 5,81 & 11,48 \\
\hline Myrcia cf. lingua (O. Berg) Mattos \& Legrand & MYRTACEAE & 77 & 3,16 & 3,56 & 1,35 & 8,07 \\
\hline Neea theifera Oerst. & NYCTAGINACEAE & 49 & 2,33 & 2,31 & 2,24 & 6,88 \\
\hline Eugenia dysenterica DC. & MYRTACEAE & 49 & 2,33 & 2,49 & 1,94 & 6,76 \\
\hline Annona coriacea Mart. & ANNONACEAE & 56 & 2,66 & 2,67 & 1,42 & 6,75 \\
\hline Caryocar brasiliense Camb. & CARYOCARACEAE & 28 & 1,33 & 1,42 & 3,80 & 6,55 \\
\hline Schefflera macrocarpum Seem. & ARALIACEAE & 56 & 2,31 & 2,67 & 1,16 & 6,14 \\
\hline Virola sebifera Aubl. & MYRISTICACEAE & 25 & 1,16 & 1,24 & 3,34 & 5,74 \\
\hline Xylopia aromatica (Lam.) Mart. & ANNONACEAE & 42 & 2,00 & 1,78 & 1,79 & 5,57 \\
\hline Rapanea ferruginea (Ruíz e Pav.) Mez & MYRSINACEAE & 42 & 2,00 & 2,13 & 0,98 & 5,11 \\
\hline Ouratea hexasperma (A. St.-Hil.) Benth. & OCHNACEAE & 32 & 1,50 & 1,60 & 1,78 & 4,88 \\
\hline Miconia sellowiana Naudin & MELASTOMATACEAE & 21 & 1,00 & 0,89 & 2,78 & 4,67 \\
\hline Siparuna guianensis Aubl. & MONIMIACEAE & 35 & 1,66 & 1,78 & 1,19 & 4,63 \\
\hline Symplocos cf. Pubescens Klotzsch ex Benth. & SYMPLOCACEAE & 21 & 1,00 & 1,06 & 1,98 & 4,04 \\
\hline Pouteria ramiflora (Mart.) Radlk. & SAPOTACEAE & 32 & 1,50 & 1,60 & 0,65 & 3,75 \\
\hline Licania humilis Cham e Schlect & CHRYSOBALANACEAE & 25 & 1,16 & 1,24 & 1,27 & 3,67 \\
\hline Styrax ferrugineus Nees e Mart. & STYRACACEAE & 28 & 1,33 & 1,42 & 0,60 & 3,35 \\
\hline Kielmeyera coriacea (Spreng.) Mart. & CLUSIACEAE & 28 & 1,33 & 1,42 & 0,57 & 3,32 \\
\hline Miconia langsdorffii Cogn. & MELASTOMATACEAE & 28 & 1,33 & 1,42 & 0,56 & 3,31 \\
\hline Senna rugosa (G. Don) Irwin e Barneby & CAESALPINIACEAE & 28 & 1,33 & 1,42 & 0,34 & 3,09 \\
\hline Myrcia rostrata DC. & MYRTACEAE & 25 & 1,16 & 1,06 & 0,86 & 3,08 \\
\hline Diospyros hispida A. DC. & EBENACEAE & 25 & 1,16 & 1,24 & 0,64 & 3,04 \\
\hline Acosmium dasycarpum (Vogel) Yakovlev & FABACEAE & 21 & 1,00 & 1,06 & 0,98 & 3,04 \\
\hline Tabebuia aurea (Mart.) & BIGNONIACEAE & 25 & 1,16 & 1,24 & 0,48 & 2,88 \\
\hline Plenckia populnea (Reiss) Lund. & CELASTRACEAE & 18 & 0,83 & 0,89 & 0,76 & 2,48 \\
\hline Guapira noxia (Netto) Lundell & NYCTAGINACEAE & 21 & 1,00 & 1,06 & 0,39 & 2,45 \\
\hline Acosmium subelegans (Mohlenbr.) Yakovlev & FABACEAE & 18 & 0,83 & 0,89 & 0,66 & 2,38 \\
\hline Qualea multiflora Mart. & VOCHYSIACEAE & 18 & 0,83 & 0,89 & 0,55 & 2,27 \\
\hline Aspidosperma tomentosum Mart. & APOCYNACEAE & 11 & 0,50 & 0,53 & 0,59 & 1,62 \\
\hline Hancornia speciosa Gomez & APOCYNACEAE & 14 & 0,66 & 0,71 & 0,17 & 1,54 \\
\hline Callisthene major Mart. & VOCHYSIACEAE & 11 & 0,50 & 0,53 & 0,45 & 1,48 \\
\hline Roupala montana Aubl. & PROTEACEAE & 7 & 0,33 & 0,35 & 0,76 & 1,44 \\
\hline Simarouba versicolor A. St.-Hil. & SIMAROUBACEAE & 11 & 0,50 & 0,53 & 0,18 & 1,21 \\
\hline Brosimum gaudichaudii Trécul & MORACEAE & 7 & 0,33 & 0,35 & 0,46 & 1,14 \\
\hline Tapirira guianensis Aubl. & ANACARDIACEAE & 7 & 0,33 & 0,35 & 0,28 & 0,96 \\
\hline Annona crassiflora Mart. & ANNONACEAE & 7 & 0,33 & 0,35 & 0,25 & 0,93 \\
\hline Byrsonima verbascifolia (L.) DC. & MALPIGHIACEAE & 4 & 0,16 & 0,17 & 0,48 & 0,81 \\
\hline
\end{tabular}




\begin{tabular}{lllllll}
\hline Crescentia cujete L. & BIGNONIACEAE & 7 & 0,33 & 0,35 & 0,13 & 0,81 \\
Cybistax antisyphilitica (Mart.) Mart. ex DC. & BIGNONIACEAE & 3 & 0,33 & 0,35 & 0,05 & 0,73 \\
Solanum lycocarpum A. St.-Hil. & SOLANACEAE & 2 & 0,33 & 0,35 & 0,05 & 0,73 \\
Himatanthus obovatus (Müill. Arg.) Woodson & APOCYNACEAE & 1 & 0,16 & 0,17 & 0,25 & 0,58 \\
Protium heptaphyllum (Aubl.) March & BURSERACEAE & 1 & 0,16 & 0,17 & 0,25 & 0,58 \\
Sclerolobium paniculatum Vogel & CAESALPINIACEAE & 1 & 0,16 & 0,17 & 0,25 & 0,58 \\
Lafoensia pacari A. St.-Hil. & LYTHRACEAE & 1 & 0,16 & 0,17 & 0,25 & 0,58 \\
Casearia decandra Jacq. & FLACOURTIACEAE & 1 & 0,16 & 0,17 & 0,25 & 0,58 \\
Anacardium occidentale L. & ANARCADIACEAE & 1 & 0,16 & 0,17 & 0,25 & 0,58 \\
Sthrychnos pseudo-quina A. St.-Hil. & LOGANIACEAE & 1 & 0,16 & 0,17 & 0,25 & 0,58 \\
Hyptidendron canum (Pohl. ex. Benth.) RM. Harley & LAMIACEAE & 1 & 0,16 & 0,17 & 0,25 & 0,58 \\
Machaerium opacum Vogel & FABACEAE & 1 & 0,16 & 0,17 & 0,25 & 0,58 \\
Annona dioica A. St.-Hil. & ANNONACEAE & 1 & 0,16 & 0,17 & 0,25 & 0,58 \\
Duguetia furfuracea (A. St.-Hil.) Saff. & ANNONACEAE & 1 & 0,16 & 0,17 & 0,25 & 0,58 \\
Bauhinia holophylla Steud. & CAESALPINIACEAE & 1 & 0,16 & 0,17 & 0,25 & 0,58 \\
Cabralea canjerana (Vell.) Mart. & MELIACEAE & 1 & 0,16 & 0,17 & 0,25 & 0,58 \\
Eriotheca pubescens (Mart. e Zucc.) Schott e Endl. & MALVACEAE & 1 & 0,16 & 0,17 & 0,25 & 0,58 \\
Ocotea pulchella Mart. & LAURACEAE & 1 & 0,16 & 0,17 & 0,25 & 0,58 \\
Couepia grandiflora (Mart. e Zucc.) Benth. ex Hook. f. CHRYSOBALANACEAE & 1 & 0,16 & 0,17 & 0,25 & 0,58 \\
Não identificadas & & 7 & 1,28 & 1,36 & 1,04 & 3,68 \\
\hline
\end{tabular}

Tabela 3. Espécies, famílias e parâmetros fitossociológicos das árvores amostradas próximas ao aterro sanitário de Goiânia.

Table 3. Species, families and phytossociologic parameters of the sampled trees nearby sanitary landfill of Goiania City.

\begin{tabular}{|c|c|c|c|c|c|c|}
\hline Espécie & Família & $\mathbf{N}^{\circ}$ ind & DR & FR & DoR & VI \\
\hline Qualea parviflora Mart. & VOCHYSIACEAE & 211 & 9,63 & 4,40 & 9,18 & 24,21 \\
\hline Eugenia dysenterica DC. & MYRTACEAE & 121 & 8,74 & 4,40 & 6,04 & 20,18 \\
\hline Pera glabrata (Schott) Baill. & EUPHORBIACEAE & 98 & 4,28 & 2,29 & 12,27 & 17,91 \\
\hline Erythroxylum suberosum A. St.-Hil. & ERYTHROXYLACEAE & 91 & 6,72 & 2,95 & 7,91 & 17,35 \\
\hline Roupala montana Aubl. & PROTEACEAE & 90 & 6,72 & 3,80 & 3,57 & 13,69 \\
\hline Qualea grandiflora Mart. & VOCHYSIACEAE & 85 & 5,75 & 2,72 & 3,38 & 11,86 \\
\hline Erythroxylum daphnites Mart. & ERYTHROXYLACEAE & 92 & 3,88 & 2,72 & 3,98 & 10,59 \\
\hline Kielmeyera cf. Grandiflora (Wawra) Saddi & CLUSIACEAE & 71 & 3,06 & 2,72 & 4,43 & 10,22 \\
\hline Curatella americana $\mathrm{L}$. & DILLENIACEAE & 86 & 3,81 & 2,95 & 2,71 & 9,46 \\
\hline Schefflera macrocarpa (Seem.) Frodin & ARALIACEAE & 52 & 2,17 & 2,72 & 4,43 & 9,31 \\
\hline Xylopia aromatica (Lam.) Mart. & ANNONACEAE & 43 & 3,51 & 3,47 & 2,10 & 8,79 \\
\hline Machaerium opacum Vogel & FABACEAE & 41 & 3,58 & 3,17 & 1,93 & 8,69 \\
\hline Salvertia convallariaeodora A. St.-Hil. & VOCHYSIACEAE & 40 & 2,76 & 2,72 & 1,85 & 7,34 \\
\hline Dalbergia miscolobium Benth. & FABACEAE & 48 & 1,87 & 2,49 & 2,89 & 7,26 \\
\hline Byrsonima verbascifolia Rich. ex Juss. & MALPIGHIACEAE & 61 & 2,84 & 2,72 & 0,66 & 6,22 \\
\hline Acosmium dasycarpum (Vogel) Yakovlev & FABACEAE & 49 & 1,94 & 2,27 & 1,85 & 6,06 \\
\hline Annona crassiflora Mart. & ANNONACEAE & 25 & 1,87 & 2,27 & 0,96 & 5,09 \\
\hline Hyptis cana Pohl ex Benth. & LAMIACEAE & 39 & 1,64 & 2,04 & 1,13 & 4,82 \\
\hline Magonia pubescens A. St.-Hil. & SAPINDACEAE & 27 & 1,87 & 2,27 & 0,64 & 4,78 \\
\hline Plathymenia reticulata Benth. & MIMOSACEAE & 31 & 1,05 & 1,94 & 1,65 & 4,51 \\
\hline Byrsonima coccolobifolia H.B.K. & MALPIGHIACEAE & 15 & 1,12 & 1,59 & 1,77 & 4,48 \\
\hline Rudgea viburnoides (Cham.) Benth. & RUBIACEAE & 19 & 1,12 & 1,59 & 1,69 & 4,40 \\
\hline Caryocar brasiliense Cambess. & CARYOCARACEAE & 19 & 0,67 & 1,36 & 2,24 & 4,27 \\
\hline Terminalia argentea Mart. \& Zucc. & COMBRETACEAE & 16 & 1,12 & 1,81 & 1,06 & 4,00 \\
\hline Erythroxylum deciduum A. St.-Hil. & ERYTHROXYLACEAE & 13 & 0,97 & 2,04 & 0,90 & 3,92 \\
\hline Dimorphandra mollis Benth. & CAESALPINIACEAE & 18 & 2,17 & 0,91 & 0,48 & 3,56 \\
\hline Piptocarpha rotundifolia (Less.) Baker & ASTERACEAE & 19 & 0,67 & 1,13 & 1,62 & 3,42 \\
\hline Tapirira guianensis Aubl. & ANACARDIACEAE & 13 & 0,45 & 0,45 & 2,22 & 3,12 \\
\hline Myrcia lingua $(\mathrm{O}$. Berg) Mattos \& D. Legrand. & MYRTACEAE & 17 & 1,12 & 1,36 & 0,56 & 3,04 \\
\hline Bowdichia virgilioides H.B.K. & FABACEAE & 13 & 0,97 & 1,36 & 0,64 & 2,97 \\
\hline Alibertia sessilis (Vell.) K. Schum. & RUBIACEAE & 10 & 0,67 & 1,36 & 0,90 & 2,94 \\
\hline Stryphnodendron adstringens (Mart.) Coville & CAESALPINIACEAE & 11 & 0,67 & 1,81 & 0,24 & 2,73 \\
\hline Styrax camporum Pohl & STYRACACEAE & 11 & 0,37 & 0,91 & 1,43 & 2,71 \\
\hline Zeyheria digitalis (Vell.) Hoehne \& Kuhlm. & BIGNONIACEAE & 10 & 0,67 & 1,59 & 0,35 & 2,61 \\
\hline Diospyros hispida A. DC. & EBENACEAE & 7 & 0,60 & 1,59 & 0,35 & 2,53 \\
\hline
\end{tabular}




\begin{tabular}{|c|c|c|c|c|c|c|}
\hline Guapira noxia (Netto) Lundell & NYCTAGINACEAE & 8 & 0,60 & 1,13 & 0,71 & 2,44 \\
\hline Erythroxylum tortuosum Mart. & ERYTHROXYLACEAE & 5 & 0,52 & 1,13 & 0,61 & 2,27 \\
\hline Sclerolobium paniculatum Vogel & CAESALPINIACEAE & 4 & 0,30 & 0,91 & 0,91 & 2,11 \\
\hline Hymenaea stigonocarpa Mart. & CAESALPINIACEAE & 2 & 0,45 & 1,36 & 0,28 & 2,08 \\
\hline Qualea multiflora Mart. & VOCHYSIACEAE & 3 & 0,45 & 0,91 & 0,37 & 1,72 \\
\hline Astronium fraxinifolium Schott & ANACARDIACEAE & 2 & 0,45 & 0,91 & 0,35 & 1,71 \\
\hline Myrsine guianensis (Aubl.) Kuntze & MYRSINACEAE & 3 & 0,45 & 0,91 & 0,32 & 1,67 \\
\hline Symplocos nitens (Pohl) Benth. & SYMPLOCACEAE & 4 & 0,30 & 0,91 & 0,42 & 1,62 \\
\hline Tabebuia aurea (Silva Manso) Benth. \& Hook.f. ex S. & & & & & & \\
\hline Moore & BIGNONIACEAE & 2 & 0,22 & 0,45 & 0,91 & 1,59 \\
\hline Agonandra brasiliensis Benth. \& Hook. f. & OPILIACEAE & 1 & 0,52 & 0,91 & 0,15 & 1,58 \\
\hline Siparuna guianensis Aubl. & MONIMIACEAE & 6 & 0,15 & 0,45 & 0,95 & 1,55 \\
\hline Enterolobium gummiferum (Mart.) J. F. Macbr. & MIMOSACEAE & 4 & 0,29 & 0,91 & 0,32 & 1,52 \\
\hline Miconia albicans (Sw.) Triana & MELASTOMATACEAE & 1 & 0,37 & 0,68 & 0,41 & 1,46 \\
\hline Aspidosperma tomentosum Mart. & APOCYNACEAE & 1 & 0,22 & 0,45 & 0,69 & 1,36 \\
\hline Strychnos pseudoquina A. St.-Hil. & LOGANIACEAE & 1 & 0,30 & 0,91 & 0,13 & 1,33 \\
\hline Palicourea rigida Kunth & RUBIACEAE & 1 & 0,30 & 0,68 & 0,18 & 1,16 \\
\hline Myrcia cf. formosiana DC. & MYRTACEAE & 1 & 0,22 & 0,68 & 0,19 & 1,09 \\
\hline Tabebuia ochracea (Cham.) Standl. & BIGNONIACEAE & 1 & 0,22 & 0,45 & 0,36 & 1,04 \\
\hline Myrcia tomentosa (Aubl.) DC. & MYRTACEAE & 1 & 0,30 & 0,68 & 0,05 & 1,03 \\
\hline Vochysia rufa Mart. & VOCHYSIACEAE & 1 & 0,15 & 0,45 & 0,26 & 0,86 \\
\hline Qualea cordata Spreng. & VOCHYSIACEAE & 1 & 0,06 & 0,79 & 0,33 & 0,86 \\
\hline Protium heptaphyllum (Aubl.) Marchand & BURSERACEAE & 1 & 0,22 & 0,45 & 0,06 & 0,74 \\
\hline Syagrus flexuosa (Mart.) Becc. & ARECACEAE & 1 & 0,22 & 0,45 & 0,05 & 0,72 \\
\hline Platypodium elegans Vogel & FABACEAE & 1 & 0,22 & 0,45 & 0,03 & 0,71 \\
\hline Plenckia populnea (Reissek) Lundell & CELASTRACEAE & 1 & 0,15 & 0,45 & 0,05 & 0,66 \\
\hline Tibouchina aegopogon (Naud.) Cogn. & MELASTOMATACEAE & 1 & 0,15 & 0,45 & 0,05 & 0,65 \\
\hline Copaifera langsdorffii Desf. & CAESALPINIACEAE & 1 & 0,15 & 0,45 & 0,04 & 0,64 \\
\hline Ouratea spectabilis (Mart.) Engl. & OCHNACEAE & 1 & 0,15 & 0,45 & 0,02 & 0,63 \\
\hline Connarus suberosus Planch. & CONNARACEAE & 1 & 0,07 & 0,23 & 0,25 & 0,55 \\
\hline Vanillosmopsis erythropappa Sch. Bip. & ASTERACEAE & 1 & 0,07 & 0,23 & 0,19 & 0,49 \\
\hline Tabebuia sp. & BIGNONIACEAE & 1 & 0,15 & 0,23 & 0,10 & 0,48 \\
\hline Antonia ovata Pohl & LOGANIACEAE & 1 & 0,15 & 0,23 & 0,05 & 0,43 \\
\hline Miconia ligustroides (DC.) Naudin & MELASTOMATACEAE & 1 & 0,07 & 0,23 & 0,12 & 0,42 \\
\hline Pseudobombax longiflorum (Mart. Zucc.) A. Robyns & MALVACEAE & 1 & 0,07 & 0,23 & 0,11 & 0,41 \\
\hline Baccharis intermixta Gardn & ASTERACEAE & 1 & 0,07 & 0,23 & 0,06 & 0,36 \\
\hline Tocoyena formosa (Cham. e Schltdl.) K. Schum. & RUBIACEAE & 1 & 0,07 & 0,23 & 0,05 & 0,35 \\
\hline Heteropterys byrsonimifolia A. Juss. & MALPIGHIACEAE & 1 & 0,07 & 0,23 & 0,04 & 0,34 \\
\hline Lafoensia pacari A. St.-Hil. & LYTHRACEAE & 1 & 0,07 & 0,23 & 0,03 & 0,33 \\
\hline
\end{tabular}

Tabela 4. Espécies, famílias e parâmetros fitossociológicos das árvores amostradas na Universidade Católica de Goiás (área-controle).

Table 4. Species, families and phytossociologic parameters of the sampled trees at Catholic University of Goias State (control area).

\begin{tabular}{|c|c|c|c|c|c|c|}
\hline Espécie & Família & $\mathbf{N}^{\circ}$ ind & DR & FR & DoR & VI \\
\hline Hymenaea stigonocarpa Mart. & CAESALPINIACEAE & 319 & 13,79 & 9,19 & 9,71 & 32,69 \\
\hline Byrsonima verbascifolia (L.) Rich. Ex A. Juss. & MALPIGHIACEAE & 117 & 4,27 & 3,73 & 3,88 & 11,87 \\
\hline Caryocar brasiliense Cambess. & CARYOCARACEAE & 94 & 5,07 & 4,10 & 4,28 & 13,45 \\
\hline Dalbergia miscolobium Benth. & FABACEAE & 151 & 8,04 & 5,50 & 5,76 & 19,30 \\
\hline Diospyros híspida A. DC. & EBENACEAE & 124 & 6,65 & 4,85 & 5,07 & 16,57 \\
\hline Hyptis cana Pohl ex Benth. & LAMIACEAE & 109 & 5,84 & 3,47 & 3,60 & 12,90 \\
\hline Eugenia dysenterica DC. & MYRTACEAE & 98 & 5,26 & 3,20 & 3,31 & 11,77 \\
\hline Connarus suberosus Planch. & CONNARACEAE & 89 & 3,82 & 3,52 & 3,65 & 10,99 \\
\hline Copaifera langsdorffii Desf. & CAESALPINIACEAE & 82 & 3,45 & 3,35 & 3,47 & 10,27 \\
\hline Curatella americana $\mathrm{L}$. & DILLENIACEAE & 76 & 4,14 & 3,67 & 3,82 & 11,63 \\
\hline Erythroxylum daphnites Mart. & ERYTHROXYLACEAE & 71 & 3,88 & 2,55 & 2,61 & 9,04 \\
\hline Miconia ligustroides (DC.) Naudin & MELASTOMATACEAE & 67 & 3,64 & 2,44 & 2,50 & 8,57 \\
\hline Syagrus flexuosa (Mart.) Becc. & ARECACEAE & 63 & 2,43 & 1,87 & 1,89 & 6,19 \\
\hline Ouratea spectabilis (Mart.) Engl. & OCHNACEAE & 59 & 3,24 & 2,25 & 2,30 & 7,78 \\
\hline Magonia pubescens A. St.-Hil. & SAPINDACEAE & 56 & 2,07 & 1,70 & 1,71 & 5,47 \\
\hline Kielmeyera grandiflora (Wawra) Saddi & CLUSIACEAE & 53 & 2,91 & 2,09 & 2,13 & 7,12 \\
\hline
\end{tabular}

FLORESTA, Curitiba, PR, v. 40, n. 1, p. 93-110, jan./mar. 2010. 


\begin{tabular}{|c|c|c|c|c|c|c|}
\hline Lafoensia pacari A. St.-Hil. & LYTHRACEAE & 17 & 1,03 & 1,21 & 1,19 & 3,44 \\
\hline Byrsonima coccolobifolia H.B.K. & MALPIGHIACEAE & 16 & 0,99 & 1,19 & 1,17 & 3,34 \\
\hline Bowdichia virgilioides H.B.K. & FABACEAE & 15 & 0,94 & 1,17 & 1,15 & 3,26 \\
\hline Myrcia lingua Berg & MYRTACEAE & 14 & 0,90 & 1,15 & 1,13 & 3,18 \\
\hline Myrsine guianensis (Aubl.) Kuntze & MYRSINACEAE & 13 & 0,86 & 1,13 & 1,11 & 3,11 \\
\hline Erythroxylum suberosum A. St.-Hil. & ERYTHROXYLACEAE & 13 & 0,83 & 1,12 & 1,09 & 3,03 \\
\hline Eremanthus glomerulatus Less. & ASTERACEAE & 12 & 0,79 & 1,10 & 1,07 & 2,97 \\
\hline Byrsonima coccolobifolia H.B.K. & MALPIGHIACEAE & 11 & 0,76 & 1,08 & 1,06 & 2,90 \\
\hline Dimorphandra mollis Benth. & CAESALPINIACEAE & 11 & 0,73 & 1,07 & 1,04 & 2,84 \\
\hline Terminalia argentea Mart. & COMBRETACEAE & 10 & 0,70 & 1,06 & 1,03 & 2,78 \\
\hline Annona crassiflora Mart. & ANNONACEAE & 10 & 0,67 & 1,04 & 1,01 & 2,73 \\
\hline Heteropterys byrsonimifolia A. Juss. & MALPIGHIACEAE & 9 & 0,65 & 1,03 & 1,00 & 2,67 \\
\hline Acosmium dasycarpum (Vogel) Yakovlev & FABACEAE & 9 & 0,62 & 1,02 & 0,98 & 2,62 \\
\hline Agonandra brasiliensis Benth. \& Hook. f. & OPILIACEAE & 8 & 0,59 & 1,01 & 0,97 & 2,57 \\
\hline Erythroxylum tortuosum Mart. & ERYTHROXYLACEAE & 8 & 0,57 & 0,99 & 0,96 & 2,53 \\
\hline Guapira noxia (Netto) Lundell & NYCTAGINACEAE & 7 & 0,55 & 0,98 & 0,95 & 2,48 \\
\hline Alibertia sessilis (Vell.) K. Schum. & RUBIACEAE & 7 & 0,52 & 0,97 & 0,94 & 2,43 \\
\hline \multicolumn{7}{|c|}{ Tabebuia aurea (Silva Manso) Benth. \& Hook.f. ex } \\
\hline S. Moore & BIGNONIACEAE & 6 & 0,50 & 0,96 & 0,93 & 2,39 \\
\hline Styrax camporum Pohl & STYRACACEAE & 6 & 0,48 & 0,95 & 0,92 & 2,35 \\
\hline Qualea cordata Spreng. & VOCHYSIACEAE & 6 & 0,46 & 0,94 & 0,91 & 2,31 \\
\hline Zeyheria digitalis (Vell.) Hoehne \& Kuhlm. & BIGNONIACEAE & 5 & 0,55 & 0,99 & 0,95 & 2,49 \\
\hline Symplocos nitens (Pohl) Benth. & SYMPLOCACEAE & 5 & 0,42 & 0,93 & 0,89 & 2,23 \\
\hline Tabebuia ochracea (Cham.) Stand. & BIGNONIACEAE & 4 & 0,40 & 0,92 & 0,88 & 2,19 \\
\hline Erythroxylum deciduum A. St.-Hil. & ERYTHROXYLACEAE & 4 & 0,38 & 0,91 & 0,87 & 2,16 \\
\hline Xylopia aromatica (Lam.) Mart. & ANNONACEAE & 4 & 0,37 & 0,90 & 0,86 & 2,12 \\
\hline Qualea grandiflora Mart. & VOCHYSIACEAE & 3 & 0,35 & 0,89 & 0,85 & 2,09 \\
\hline Pera glabrata (Schott) Baill. & EUPHORBIACEAE & 3 & 0,33 & 0,88 & 0,84 & 2,06 \\
\hline Vochysia rufa Mart. & VOCHYSIACEAE & 3 & 0,32 & 0,88 & 0,83 & 2,02 \\
\hline Qualea multiflora Mart. & VOCHYSIACEAE & 2 & 0,30 & 0,87 & 0,82 & 1,99 \\
\hline Miconia cuspidata Naud. & MELASTOMATACEAE & 2 & 0,28 & 0,86 & 0,82 & 1,96 \\
\hline Piptocarpha rotundifolia (Less.) Baker & ASTERACEAE & 2 & 0,27 & 0,85 & 0,81 & 1,93 \\
\hline Tocoyena formosa (Cham. e Schltdl.) K. Schum. & RUBIACEAE & 2 & 0,25 & 0,85 & 0,80 & 1,90 \\
\hline Stryphnodendron adstringens (Mart.) Coville & MIMOSACEAE & 1 & 0,22 & 0,83 & 0,79 & 1,84 \\
\hline Tapirira guianensis Aubl. & ANACARDIACEAE & 3 & 0,33 & 0,88 & 0,84 & 2,04 \\
\hline Schefflera macrocarpa (Seem.) Frodin & ARALIACEAE & 2 & 0,29 & 0,86 & 0,82 & 1,98 \\
\hline Qualea parviflora Mart. & VOCHYSIACEAE & 1 & 0,22 & 0,83 & 0,79 & 1,84 \\
\hline Protium heptaphyllum (Aubl.) Marchand & BURSERACEAE & 1 & 0,22 & 0,83 & 0,79 & 1,84 \\
\hline Roupala montana Aubl. & PROTEACEAE & 1 & 0,22 & 0,83 & 0,79 & 1,84 \\
\hline Sclerolobium paniculatum Vogel & CAESALPINIACEAE & 1 & 0,22 & 0,83 & 0,79 & 1,84 \\
\hline Plathymenia reticulata Benth. & MIMOSACEAE & 2 & 0,27 & 0,86 & 0,81 & 1,94 \\
\hline Platypodium elegans Vogel & FABACEAE & 1 & 0,22 & 0,83 & 0,79 & 1,84 \\
\hline Rudgea viburnoides (Cham.) Benth. & RUBIACEAE & 1 & 0,22 & 0,83 & 0,79 & 1,84 \\
\hline Salvertia convallariaeodora A. St.-Hil. & VOCHYSIACEAE & 1 & 0,22 & 0,83 & 0,79 & 1,84 \\
\hline Pseudobombax longiflorum (Mart. Zucc.) A. Rob & MALVACEAE & 1 & 0,22 & 0,83 & 0,79 & 1,84 \\
\hline Machaerium opacum Vogel & FABACEAE & 1 & 0,22 & 0,83 & 0,79 & 1,84 \\
\hline
\end{tabular}

O esforço amostral foi representado pela curva espécie-área de cada subparcela amostrada. Para as quatro áreas estabelecidas (Figura 2), o esforço amostral foi suficiente. Estabilizaram-se as curvas a partir das últimas subparcelas, com alta significância nos parâmetros do ajuste da curva: $R^{2}>0,88$, $\mathrm{p}<0,001$ e $\varepsilon<0,160$ (Tabela 6).

As famílias com maiores números de indivíduos entre as quatro áreas de estudo apresentaram de 300 a 600 indivíduos, e não houve padrões de distribuição das espécies pelas família entre as áreas. A família Malpighiaceae obteve destaque por estar nas quatro áreas de estudo e entre as dez famílias com maior número de indivíduos. Melastomataceae, Fabaceae e Myrtaceae estiveram em três áreas e entre as 10 famílias com maior número de indivíduos.

Nas parcelas próximas aos aterros, foram encontradas espécies descritas principalmente como de mata de galeria, como é o caso de Cabralea cangerana e Campomanesia xanthocarpa, em Brasília, e Erythroxylum daphnites e Pera glabrata, em Goiânia (MENDONÇA et al., 1998). Segundo Marimon Júnior; Haridasan (2005), espécies típicas de ambientes florestais encontradas em ambientes de Cerrado 
típico podem ser bioindicadoras de solos mais férteis e mais úmidos nos locais em que foram encontradas, e isto pode representar a influência do aterro no solo.

Tabela 5. Número de espécies e famílias encontradas em áreas de Cerrado stricto sensu neste estudo e na literatura.

Table 5. Number of the species and families sampled in Cerrado stricto sensu areas, in this study and literature.

\begin{tabular}{llcc}
\hline Fontes & Espécies & Famílias \\
\hline Salles; Schiavini, 2007 & 63 & 32 \\
Balduíno et al., 2005 & 73 & 38 \\
Fiedler et al., 2004 & & 46 & 23 \\
Saporetti Jr. et al., 2003 & & 85 & 44 \\
Felfili et al., 2002 & & 80 & 34 \\
Batalha et al., 2001 & BSB & 81 & 40 \\
& BSB - Controle & 70 & 30 \\
Este estudo & GYN & 71 & 39 \\
& GYN - Controle & 73 & 36 \\
& & 61 & 30 \\
\hline
\end{tabular}
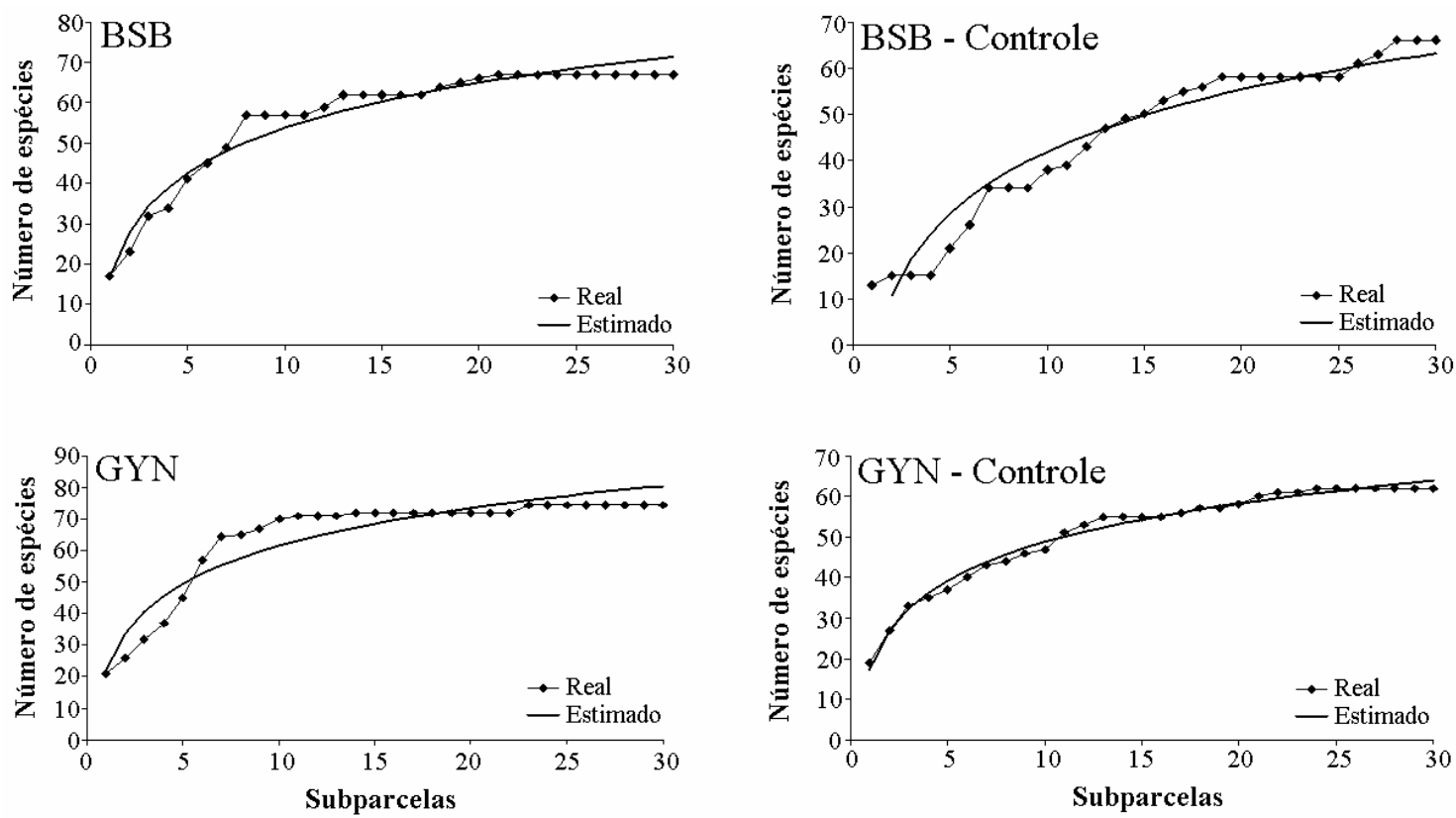

Figura 2. Curva espécie-área por subparcela amostrada.

Figure 2. Species-area curve by sampled subplot.

Tabela 6. Coeficientes e parâmetros estatísticos obtidos pela equação logarítmica da curva de espécieárea com as subparcelas amostradas.

Table 6. Coefficients and parameters statistics got by logarithm equation of the species-area curve with the sampled subplots.

\begin{tabular}{lccccc}
\hline \multicolumn{1}{c}{ Parcelas } & $\boldsymbol{\beta}_{\mathbf{0}}$ & $\boldsymbol{\beta}_{\mathbf{1}}$ & $\mathbf{R}^{\mathbf{2}}$ & $\boldsymbol{\varepsilon}$ & $\mathbf{p}$ \\
\hline BSB & 16,126 & 16,636 & 0,96 & 0,022 & $<0,001$ \\
BSB - Controle & 19,393 & $-2,761$ & 0,93 & 0,014 & $<0,001$ \\
GYN & 17,267 & 21,761 & 0,88 & 0,156 & $<0,001$ \\
GYN - Controle & 13,709 & 17,183 & 0,99 & 0,075 & $<0,001$ \\
\hline
\end{tabular}


O índice de diversidade de Shannon para este estudo apresentou valores entre 2,7 e 3,5, e o índice de Simpson, valores entre 0,86 a 0,96, corroborando osvalores médios apresentados por Felfili; Felfili (2001), de 3,3 e 0,9, respectivamente, em estudos realizados em várias áreas de Cerrado strictu sensu, utilizando metodologias semelhantes à deste estudo (Figuras 3 e 4 ).

Não houve um padrão de distribuição entre as subparcelas dos valores dos índices de diversidade. Testes estatísticos de Kruskal-Wallis aplicados entre os valores dos índices de diversidade distribuídos nas subparcelas de cada área, nas quatro áreas estudadas, não foram significativos $(\mathrm{p}>0,556)$.

Vários autores mostraram, em seus estudos, que em áreas perturbadas ou nas adjacências pode ocorrer o predomínio de uma ou poucas espécies em uma comunidade (BEGON et al., 1996; BEGON; MORTIMER, 1996; MARGALEF, 1983 e 1970). No presente estudo, observou-se que os depósitos de lixo não influenciaram na diversidade das espécies nativas nas subparcelas estudadas.
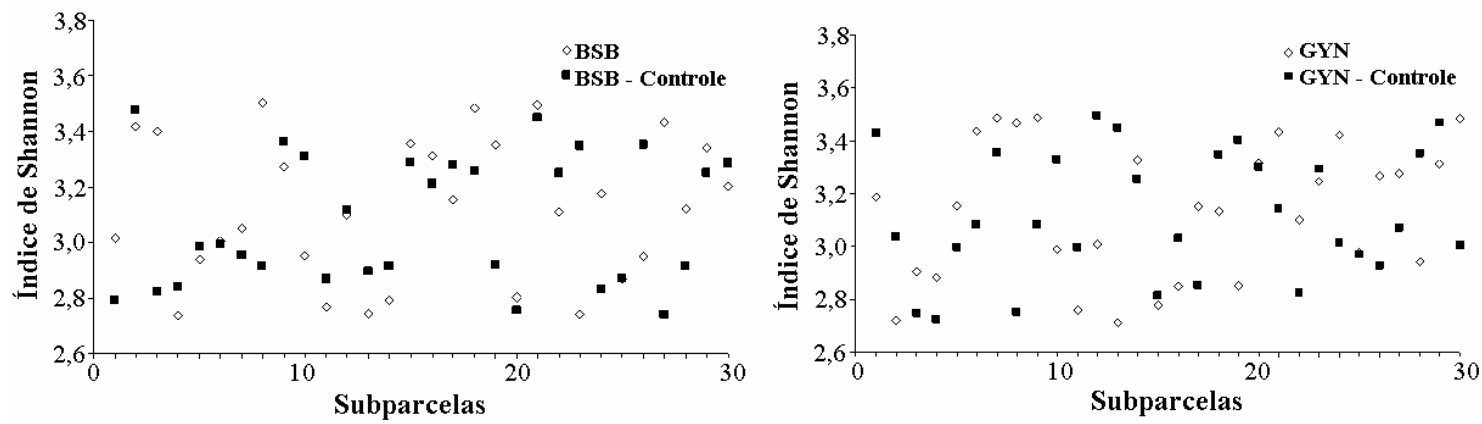

Figura 3. Índice de diversidade de Shannon distribuído nas 30 subparcelas estudadas: BSB (Parque Nacional de Brasília próximo ao aterro), BSB - Controle (Parque Nacional de Brasília distante do aterro), GYN (próximo ao aterro sanitário de Goiânia) e GYN - Controle (área da Universidade Católica de Goiás).

Figure 3. Diversity Index of Shannon dispersed in the 30 studied subplots: BSB (National Park of the Brasilia City, nearby landfill), BSB - Controle (National Park of the Brasilia City, farther landfill), GYN (nearby sanitary landfill of the Goiania City) and GYN - Controle (Catholic University of Goias State area).
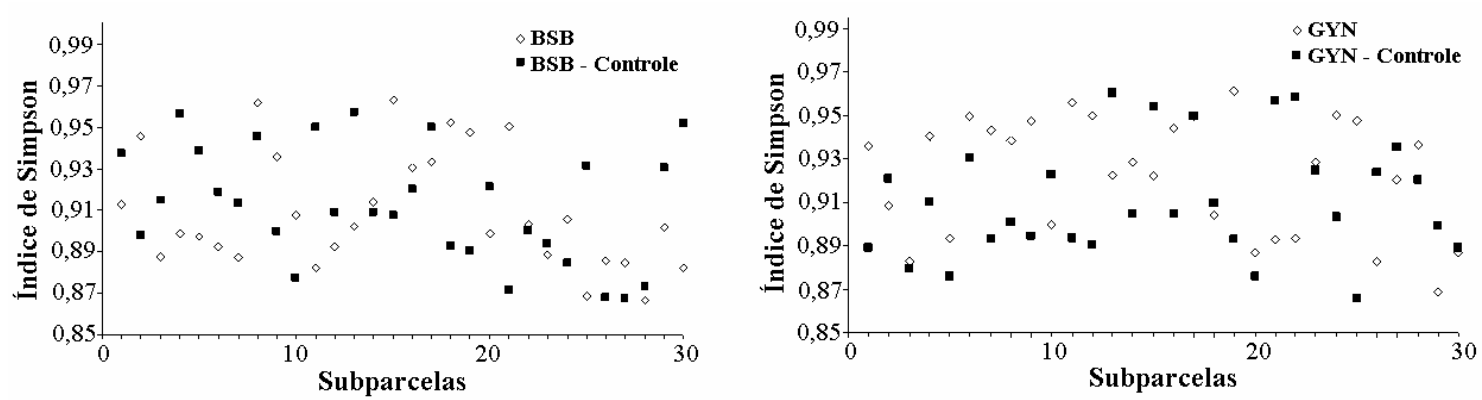

Figura 4. Índice de diversidade de Simpson distribuído nas 30 subparcelas estudadas: BSB (Parque Nacional de Brasília próximo ao aterro), BSB - Controle (Parque Nacional de Brasília distante do aterro), GYN (próximo ao aterro sanitário de Goiânia) e GYN - Controle (área da Universidade Católica de Goiás).

Figure 4. Diversity index of Simpson dispersed in the 30 studied subplots: BSB (National Park of the Brasilia City, nearby landfill), BSB - Controle (National Park of the Brasilia City, farther landfill), GYN (nearby sanitary landfill of the Goiania City) and GYN - Controle (Catholic University of Goias State area). 
A distribuição da comunidade arbórea dos indivíduos em classes diamétricas apresentou o padrão do $J$-invertido (Figura 5), ou seja, alta concentração de indivíduos nas classes menores e redução acentuada no sentido das classes maiores. Segundo Líbano; Felfili (2006) e Lima et al. (2003), a quase totalidade dos inventários de comunidades arbóreo-arbustivas de vegetação autóctones apresentam uma distribuição diamétrica seguindo o modelo do $J$-invertido (ou exponencial negativa), representando alta regeneração das espécies e distúrbios constantes (LÍBANO; FELFILI, 2006).

Os parâmetros estatísticos do ajuste da equação de Meyer dos indivíduos pelas classes diamétricas (Tabela 7) foram significativos para as quatro áreas $\left(\mathrm{R}^{2}>0,93, \varepsilon<0,180 \mathrm{e} \mathrm{p}<0,001\right)$, mostrando que os indivíduos foram distribuídos significativamente na tendência da curva de $J$-invertido.
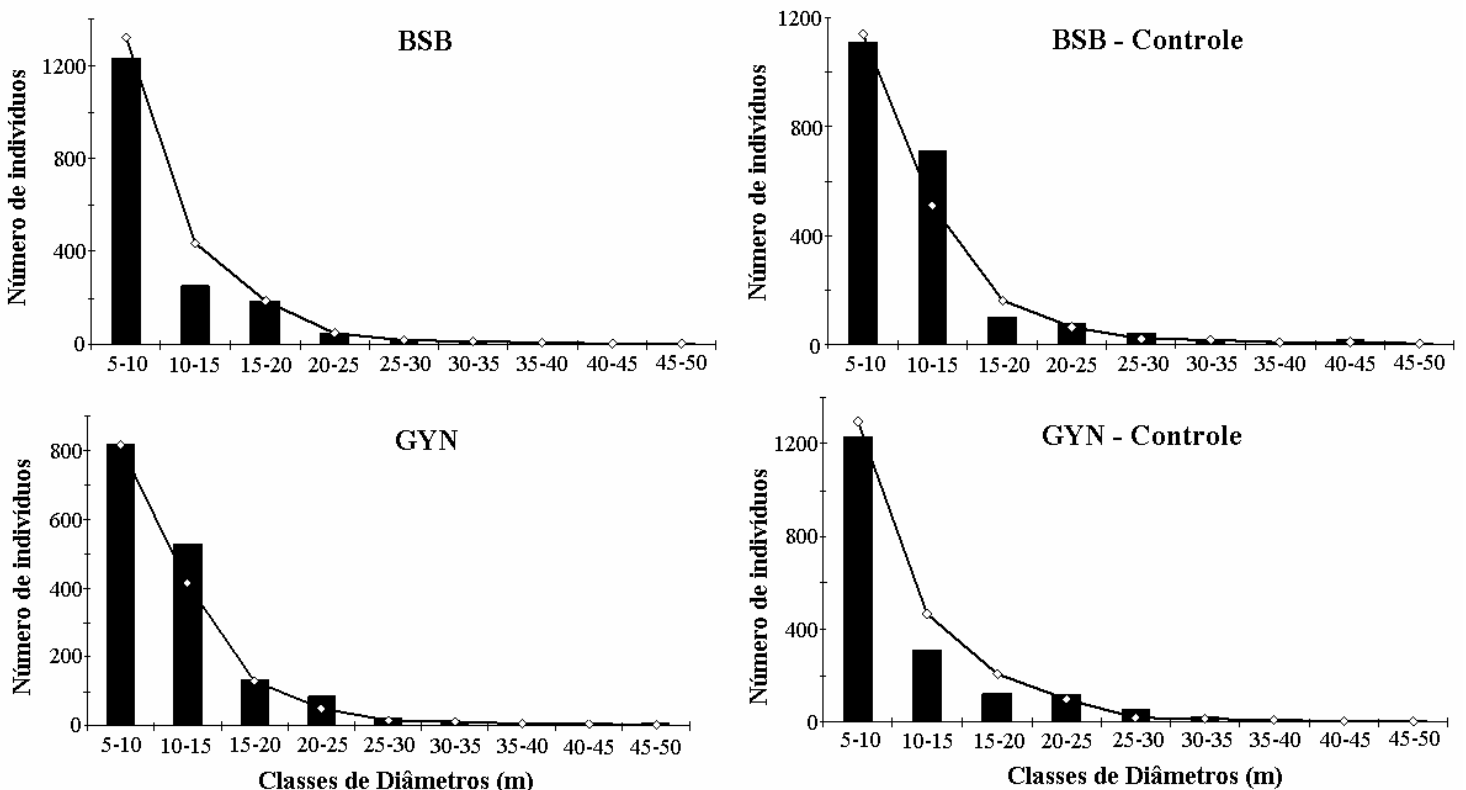

Figura 5. Distribuição em classes diamétricas dos indivíduos arbóreos nas áreas estudadas: BSB (Parque Nacional de Brasília próximo ao aterro), BSB - Controle (Parque Nacional de Brasília distante do aterro), GYN (próximo ao aterro sanitário de Goiânia) e GYN - Controle (área da Universidade Católica de Goiás). As curvas representam o ajuste exponencial de Meyer (1952).

Figure 5. Diameter class distribution of the tree individuals in studied areas: BSB (National Park of the Brasilia City, nearby landfill), BSB - Controle (National Park of the Brasilia City, farther landfill), GYN (nearby sanitary landfill of the Goiania City) and GYN - Controle (Catholic University of Goias State area). The curves are the Meyer exponential adjust (1952).

Tabela 7. Coeficientes e parâmetros estatísticos obtidos pela equação de Meyer.

Table 7. Coefficients and parameters statistics got by Meyer equation.

\begin{tabular}{lccccc}
\hline \multicolumn{1}{c}{ Parcelas } & $\boldsymbol{\beta}_{\mathbf{0}}$ & $\boldsymbol{\beta}_{\mathbf{1}}$ & $\mathbf{R}^{\mathbf{2}}$ & $\boldsymbol{\varepsilon}$ & $\mathbf{p}$ \\
\hline BSB & 1314,10 & $-0,0197$ & 0,95 & 0,175 & $<0,001$ \\
BSB - Controle & 1316,80 & $-0,0188$ & 0,96 & 0,150 & $<0,001$ \\
GYN & 1315,95 & $-0,0315$ & 0,93 & 0,164 & $<0,001$ \\
GYN - Controle & 1309,00 & $-0,0169$ & 0,98 & 0,126 & $<0,001$ \\
\hline
\end{tabular}

Os dendrogramas de ordenamento para os índices de similaridade de Morisita e Sørensen (Figuras 6, 7, 8 e 9), nas áreas estudadas, apresentaram altos valores de fusão entre as subparcelas para cada área, média de 0,645 para Morisita e 0,601 para Sørensen.

Notou-se que, nas figuras 6 a 9, as subparcelas presentes em áreas adjacentes aos aterros (representado pela letra A) apresentaram dois agrupamentos, um entre as primeiras subparcelas (da subparcela 1 a 10) e outro das subparcelas mais distantes do aterro (da subparcela 20 a 30), o que não foi observado para os dois índices nos controles. 


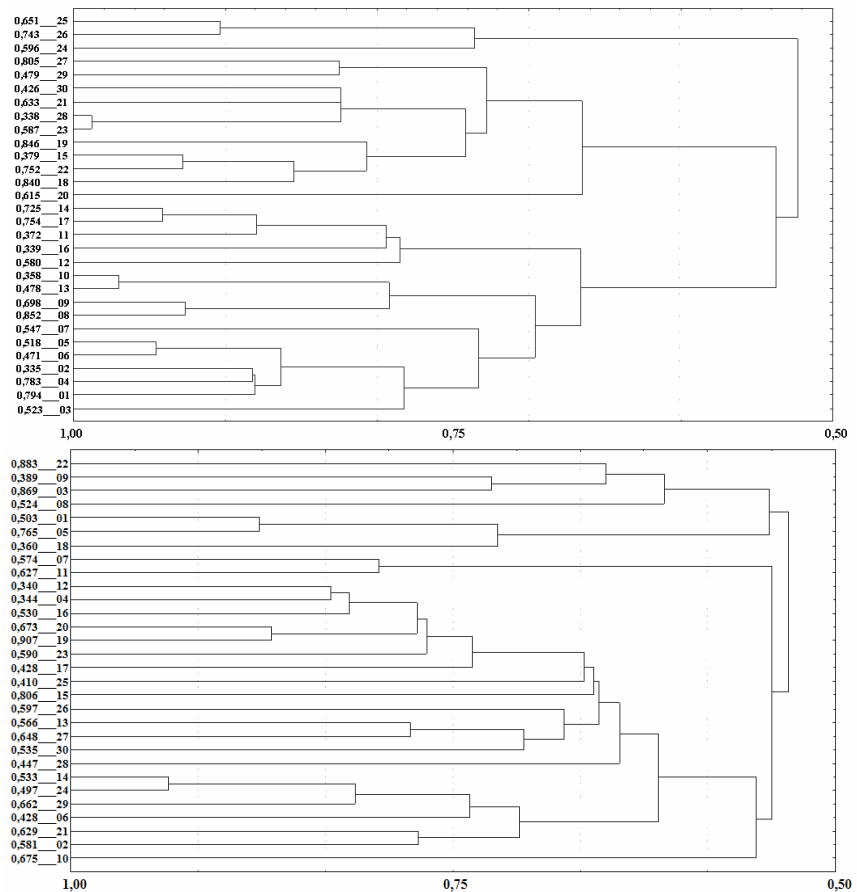

(A)

(B)

Figura 6. Classificação UPGMA (Índice de Morisita) e os níveis de fusão para as subparcelas estudadas no Parque Nacional de Brasília: (A) área mais próxima ao aterro e (B) área mais distante do aterro.

Figure 6. UPGMA classification (Morisita index) and the fusion levels to the studied subplots in National Park of Brasilia City: (A) area nearby landfill and (B) area farther of landfill.

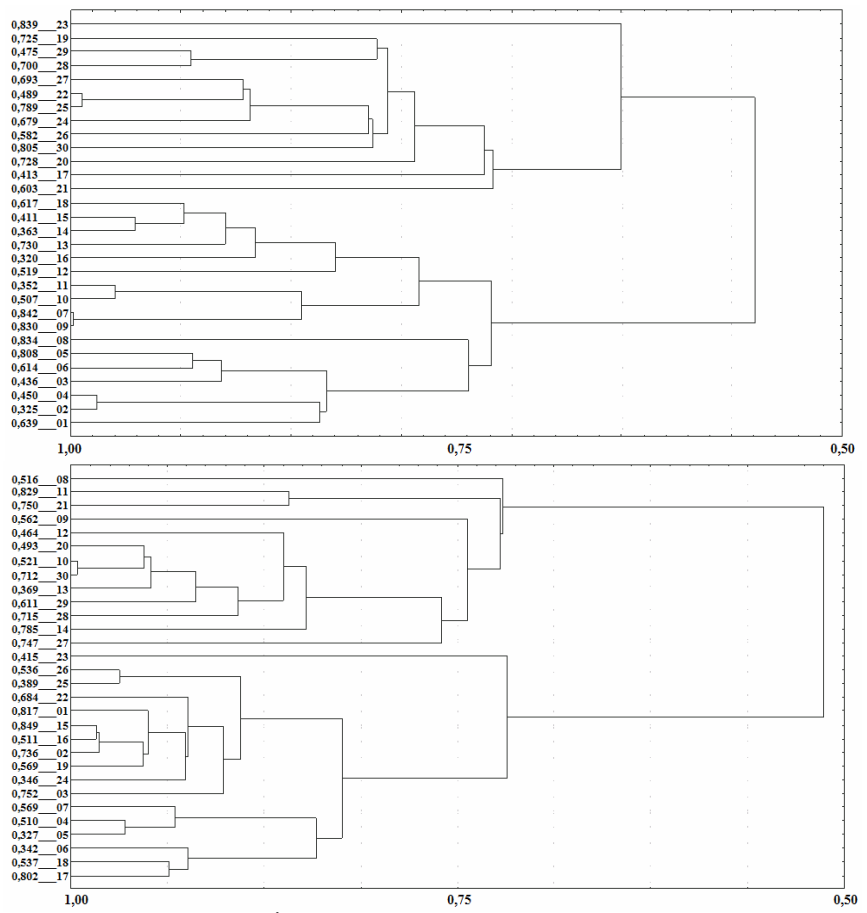

(A)

(B)

Figura 7. Classificação UPGMA (Índice de Morisita) e os níveis de fusão para as subparcelas estudadas em Goiânia: (A) área mais próxima ao aterro e (B) área mais distante do aterro.

Figure 7. UPGMA classification (Morisita index) and the fusion levels to the studied subplots in Goiania City: (A) area nearby landfill and (B) area farther of landfill. 


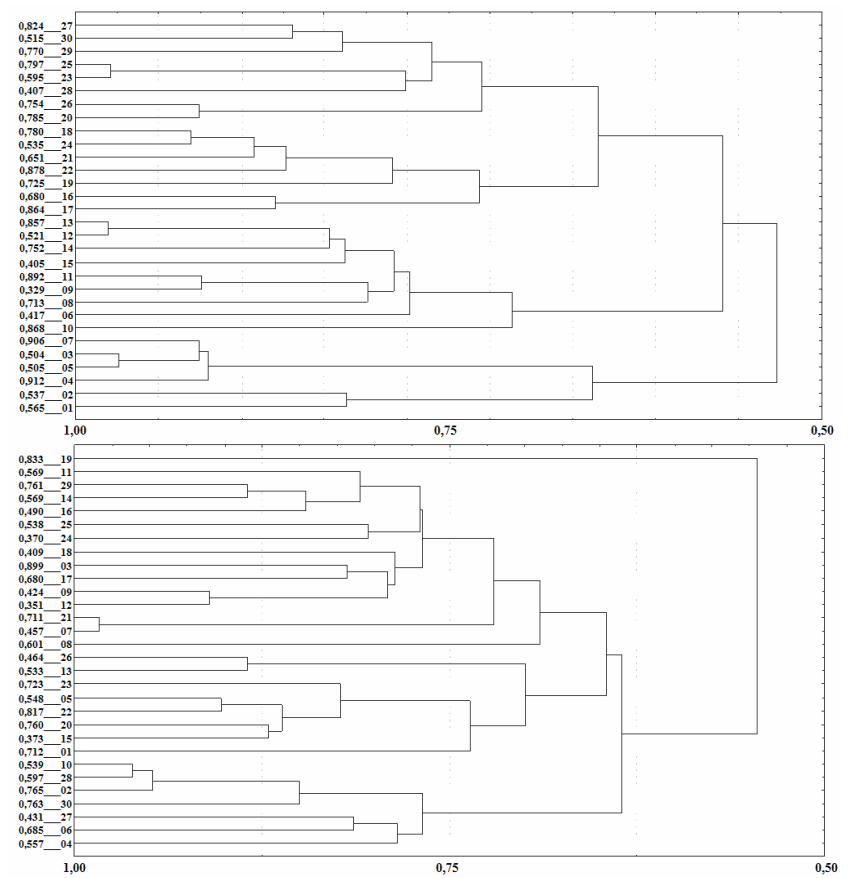

(A)

(B)

Figura 8. Classificação UPGMA (Índice de Sørensen) e os níveis de fusão para as subparcelas estudadas no Parque Nacional de Brasília: (A) área mais próxima ao aterro e (B) área mais distante do aterro.

Figure 8. UPGMA classification (Sørensen index) and the fusion levels to the studied subplots in National Park of Brasilia City: (A) area nearby landfill and (B) area farther of landfill.

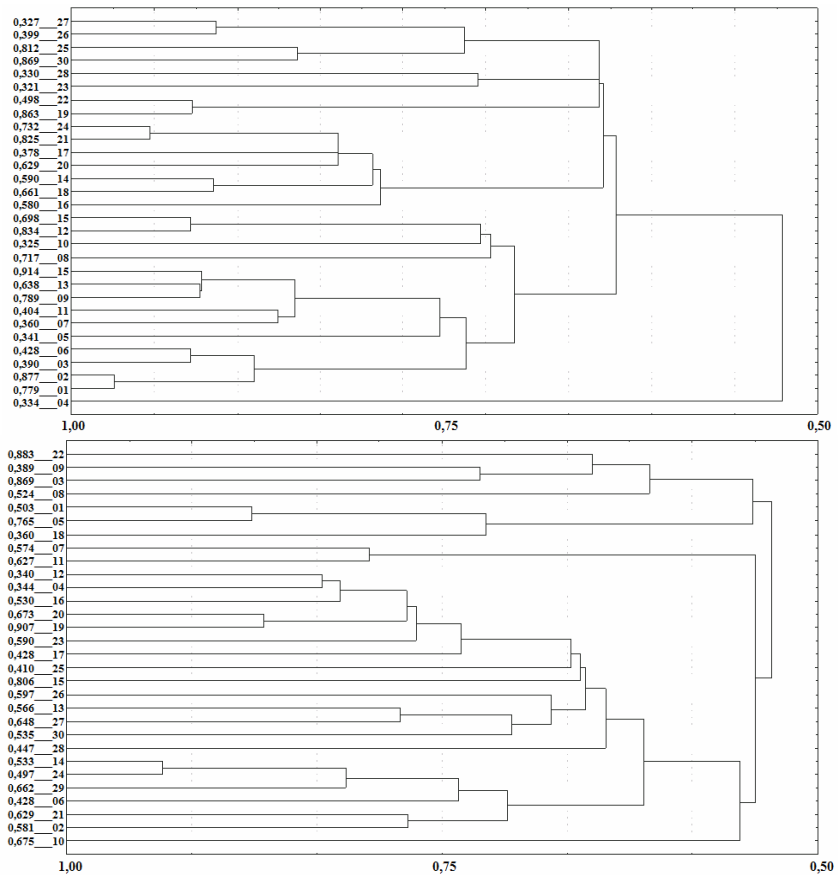

(A)

(B)

Figura 9. Classificação UPGMA (Índice de Sørensen) e os níveis de fusão para as subparcelas estudadas em Goiânia: (A) área mais próxima ao aterro e (B) área mais distante do aterro.

Figure 9. UPGMA classification (Sørensen index) and the fusion levels to the studied subplots in Goiania City: (A) area nearby landfill and (B) area farther of landfill. 
Líbano; Felfili (2006); Balduíno et al. (2005); Fonseca; Silva Jr. (2004) e Silva Jr. (2004) demonstraram esse comportamento em áreas de Cerrado, descrevendo que fatores como topografia, presença do lençol freático, classificação e análise do solo foram os principais fatores determinantes para a ordenação e agrupamento dos índices de similaridade de espécies entre as áreas. Ficou evidente que a presença do aterro foi determinante na ordenação dos níveis de fusão de similaridades entre as subparcelas, tanto pela presença e ausência de espécies, como pelo número de indivíduos encontrados nas espécies.

Observando a figura 10, quando se separou o de importância (VI) pelas subparcelas, em áreas próximas ao aterro, espécies mostraram a existência de padrões, ou seja, algumas apresentaram altos valores de VI nas proximidades dos aterros (até $200 \mathrm{~m}$ ), reduzindo esse valor a partir de subparcelas mais distantes (de 400 a $500 \mathrm{~m}$ ), como foi o caso da Curatella americana em Goiânia. Com outras espécies aconteceu o comportamento contrário, como foi o caso da Guapira noxia em Brasília. Outras espécies, pelos valores de VI, mantiveram-se indiferentes à presença do aterro, ou seja, não apresentaram um padrão na distribuição do VI ao longo dos gradientes, o mesmo acontecendo nas subparcelas presentes na área controle.

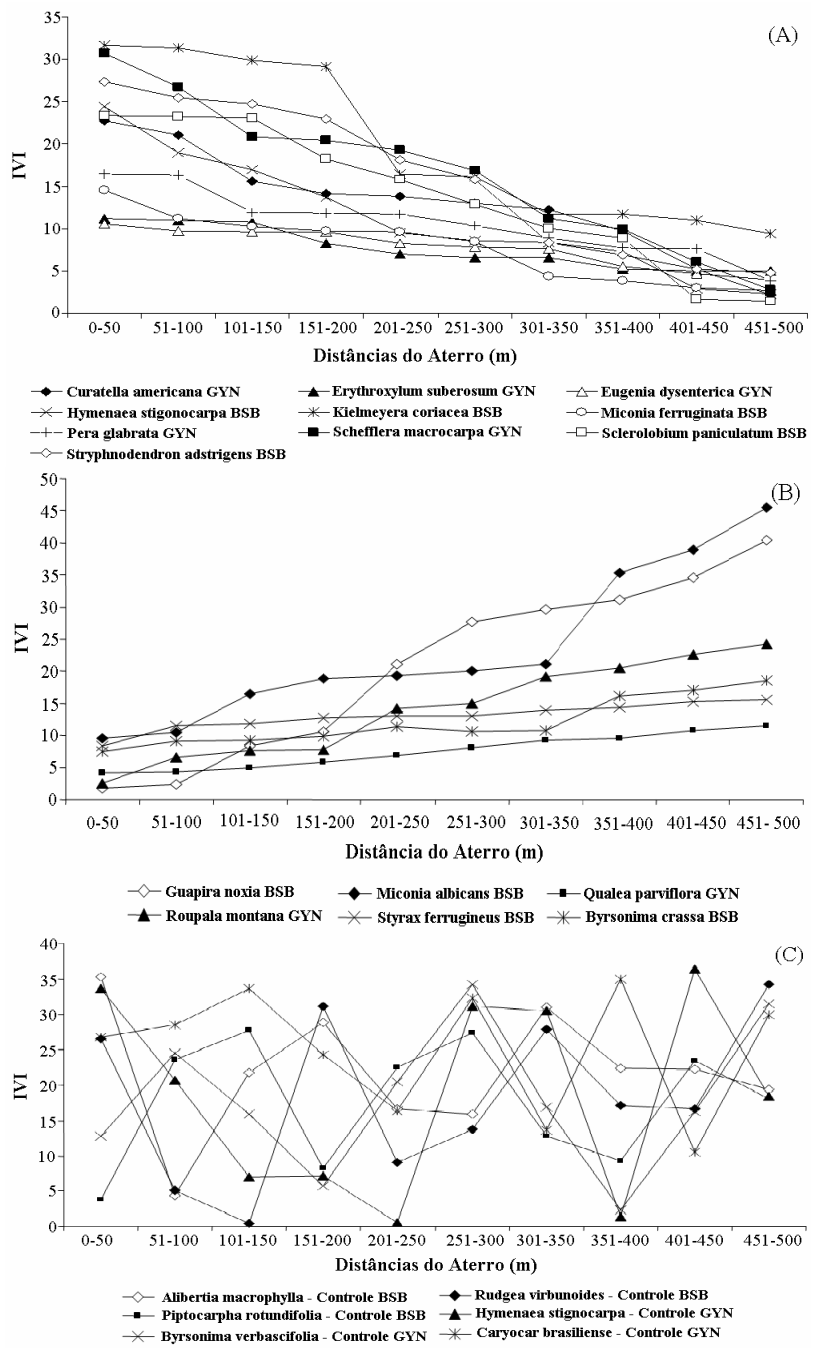

Figura 10. Valor de importância (VI) das espécies arbóreas estudadas em áreas próximas ao aterro do (A) Jockey Club de Brasília (BSB), (B) ao Aterro Sanitário de Goiânia (GYN) e (C) das áreas controles (BSB - Controle e GYN - Controle).

Figure 10. Value importance (VI) of the studied tree species in areas nearby of the landfills (A) Jockey Club of Brasilia City (BSB) and (B) Sanitary Landfill of Goiania City (GYN), and (C) of the control areas (BSB - Controle and GYN - Controle). 
Darwin et al. (2004) mostraram os padrões descritos acima, indicando que, em áreas com alguma perturbação antrópica ou natural, algumas espécies aumentaram seu VI com o passar do tempo, acontecendo o contrário com outras espécies. Silva et al. (2002), em estudos em áreas de Cerrado, observaram também esse padrão, descrevendo que a mudança na camada superficial do solo foi um fator determinante na distribuição das espécies, o que também foi confirmado por Bustamante et al. (2006); Nardoto et al. (2006) e Haridasan (2000), quando descreveram a influência da modificação dos elementos químicos do solo para os grupos funcionais (herbáceas, arbustos e árvores) da vegetação de Cerrado.

\section{CONCLUSÕES}

- A presença de aterros sanitários não influenciou na diversidade arbórea nativa do Cerrado, mas na distribuição espacial das espécies.

- A distribuição diamétrica em J-invertido não foi influenciada pela presença do aterro.

- Subparcelas próximas aos aterros são mais similares do que em áreas mais distantes, com relação tanto à presença e ausência de espécies quanto ao número de indivíduos por espécie. Padrão não observado nas áreas controles.

- Foram observados três comportamentos para distribuição dos valores de importância (VI), a partir do aterro para regiões mais distantes: i) aumento dos valores, ii) redução dos valores e iii) indiferente (nem aumento, nem redução).

\section{AGRADECIMENTOS}

Ao Parque Nacional de Brasília e ao aterro sanitário de Goiânia, pela logística. À CAPES e ao $\mathrm{CNPq}$, por fornecerem bolsa de estudo ao primeiro autor.

\section{REFERÊNCIAS}

ANDRADE, L. A. Z.; FELFILI, J. M.; VIOLATTI, L. Fitossociologia de uma área de Cerrado denso na RECOR-IBGE, Brasília-DF. Acta Botanica Brasilica, São Paulo, v. 16, n. 2, p. 225-240, 2002.

ANGIOSPERM PHYLOGENY GROUP II. An update of the angiosperm phylogeny group classification for orders and families of flowering plants: APG II. Botanical Journal of the Linnean Society, Edinburgh, v. 141, p. 399-436, 2003.

ASSUNÇÃO, S. L.; FELFILI, J. M. Fitossociologia de um fragmento de Cerrado sensu stricto na APA do Paranoá, DF, Brasil. Acta Botanica Brasilica, São Paulo, v. 18, n. 4, p. 903-909, 2004.

BALDUÍNO, A. P. C.; SOUZA, A. L.; MEIRA-NETO, J. A. A.; SILVA, A. F.; SILVA Jr, M. C. Fitossociologia e análise comparativa da composição florística do Cerrado da flora de Paraopeba-MG. Revista Árvore, Viçosa, MG, v. 29, n. 1, p. 25-34, 2005.

BATALHA, M. A.; MANTOVANI, W.; MESQUITA Jr, N. H. Vegetation structure in Cerrado physiognomies in South-eastern Brazil. Brazilian Journal of Biology, São Carlos, v. 61, n. 3, p. 475483, 2001.

BEGON, M.; HARPER, J. L.; TOWNSEND, R. C. Ecology: Individuals, populations and communities. 3. ed. Oxford, UK: Blackwell, 1996. 1068 p.

BEGON, M.; MORTIMER, M. Population ecology: a unified study of animals and plants. 2. ed. Oxford, UK: Blackwell, 1996. 220 p.

BRASÍLIA, DF. Secretaria de Estado de Desenvolvimento Urbano e Habitação (SEDUH). Disponível em: <http://www.seduh.df.gov.br/>. Acesso em: 03/07/2007.

BREYER, L. M.; TSUBOI, E. Sistema herbário: manual de operações. Brasília, DF: Editora Universidade de Brasília, 1996. 109 p. 
BUSTAMANTE, M. M. C.; MEDINA, E.; ASNER, G. P.; NARDOTO, G. B.; GARCIA-MONTIEL, D. C. Nitrogen cycling in tropical and temperate savannas. 2006. Biogeochemistry, New York, v. 79, p. 1573-1515, 2006.

CARTER, M. R. Soil sampling and methods of analysis. Boca Raton, USA: Lewis, 1993. 823 p.

CHAPMAN, S. B. Methods in Plant Ecology. The Journal of Ecology, Brighton, v. 65, n. 2, p. 718-719, 1977.

CORADIN, V. T. R.; HARIDASAN, M.; SOUZA, M. R.; SILVA, M. E. F.; PEREIRA, M. S. Influência da calagem e da adubação no crescimento de duas espécies lenhosas do Cerrado. Brasil Florestal, Brasília, DF, v. 74, p. 53-60, 2002.

COLWELL, R. K.; CODDINGTON, J. A. Estimating terrestrial biodiversity through extrapolation. Philosophical Transition of the Royal Society, Edinburgh, v. 345, p. 101-118, 1994.

DARWIN, A. T.; LADD, D.; GALDINS, R.; CONTRERAS, T. A.; FAHRIG, L. Response of forest understory vegetation to a major ice storm. Journal of Torrey Botanical Society, Bronx, v. 131, p. 45-52, 2004.

EITEN, G. Vegetação natural do Distrito Federal. Brasília, Brasil: SEBRAE, 2001. 162 p.

EMBRAPA - EMPRESA BRASILEIRA DE PESQUISA AGROPECUÁRIA. Centro Nacional de Pesquisa de Solos (Rio de Janeiro, RJ). Sistema Brasileiro de Classificação de Solos. Brasília, DF: Embrapa Produção da Informação. 1999. 412 p.

FELFILI, J. M.; NOGUEIRA, P. E.; SILVA JÚNIOR, M. C. da; MARIMON, B. S.; DELITTI, W. B. C. Composição florística e fitossociologia do Cerrado sentido restrito no município de Água Boa - MT. Acta Botanica Brasilica, São Paulo, v. 16, n. 1, p. 103-112, 2002.

FELFILI, M. C.; FELFILI, J. M. Alfa and Beta diversity in the Cerrado sensu stricto. Acta Botanica Brasilica, São Paulo, v. 15, n. 2, p. 243-254, 2001.

FIEDLER, N. C.; AZEVEDO, I. N. C. de; REZENDE, A. V.; REZENDE, A. V.; MEDEIROS, M. B.; VENTUROLI, F. Efeito de incêndios florestais na estrutura e composição florística de uma área de Cerrado sensu stricto na fazenda Água Limpa-DF. Revista Árvore, Viçosa, MG, v. 28, n. 1, p. 129-138. 2002.

FONSECA, M. S.; SILVA JÚNIOR, M. C. Fitossociologia e similaridade florística entre trechos de Cerrado sentido restrito em interflúvio e em vale no Jardim Botânico de Brasília, DF. Acta Botanica Brasilica, São Paulo, v. 18, n. 1, p. 19-29, 2004.

GOIÂNIA (GO). Prefeitura Municipal. Disponível em: http://www.goiania.go.gov.br . Acesso em: 03/07/2004.

HARIDASAN, M. Nutrição mineral de plantas nativas do Cerrado. Revista Brasileira de Fisiologia Vegetal, Campinas, v. 12, n. 1, p. 54-64, 2000.

KENT, M.; COKER, P. Vegetation description and analysis: a practical approach. New York: J. Wiley e Sons, 1992. $256 \mathrm{p}$.

KLINK, C. A.; MOREIRA, A. G. Past and current human occupation, and land use. Pp. 69-88. In: P.S. Oliveira e J.M. Robert (Eds.). The Cerrados of Brazil: ecology and natural history of a neotropical savanna. New York, USA: Columbia University Press, 2002. 256 p.

KOVACH, W. L. MVSP (Multivariate Statistical Pachage) versão 2.1. Anglesey, UK: Kovac Computing. 1993. $156 \mathrm{p}$.

LÍBANO, A. M.; FELFILI, J. M. Mudanças temporais na composição florística e na diversidade de um Cerrado sensu stricto do Brasil Central em um período de 18 anos (1985-2003). Acta Botanica Brasilica, São Paulo, v. 20, n. 4, p. 927-936, 2006. 
LIMA, E. S.; FELFILI, J. M.; MARIMON, B. S. Diversity, structure and spatial distribution of palms in a Cerrado sensu stricto in Central Brazil - DF. Revista Brasileira de Botânica, São Paulo, v. 26, n. 3, p. 361-370, 2003.

MAGURRAN, A. E. Ecological diversity and its measurement. 2. ed. Princeton: Princeton University Press, 1988. $179 \mathrm{p}$.

MARGALEF, R. 1983. Ecologia. 2. ed. Barcelona: Omega 951 p.

MARGALEF, R. 1970. Perspectives in ecological theory. Chicago: Univ Press(The) 111 p.

MARIMON JÚNIOR, B. H.; HARIDASAN, M. A comparison of the woody vegetation and soil characteristics of a cerradão and a Cerrado sensu stricto in adjacents areas on dystrophic soils in eastern Mato Grosso State, Brazil. Acta Botanica Brasilica, São Paulo, v. 19, n. 4, p. 913-926, 2005.

MENDONÇA, R. C.; FELFILI, J. M.; WALTER, B. M. T.; SILVA JÚNIOR, M. C.; REZENDE, A. V.; FILGUEIRAS, T. S.; NOGUEIRA, P. E. Flora vascular do Cerrado.. In: SANO, S. M.; ALMEIDA, S. P. Cerrado, Ambiente e flora. Planaltina: EMBRAPA/CPAC. 1998. p. 289-556

MEYER, H. A. 1952. Structure, growth and drain in balanced uneven-aged forests. Journal of Forest, Oxford, v. 50, p. 85-92, 1952.

MITTERMEIER, R. S.; MYERS, N.; GIL, P. R. Hotspots: earth's biologically richest and most endangered terrestrial ecoregions. Cidade do México: Cemex Conservation International, 1999. $50 \mathrm{p}$.

MUELLER-DOMBOIS, D.; ELLEMBERG, H. Aims and methods of vegetation ecology. New York: J. Wiley and Sons, 2002. $547 \mathrm{p}$.

NARDOTO, G. B.; BUSTAMANTE, M. M. C.; PINTO, A. S.; KLINK, C. A. Nutrient use efficiency at ecosystem and species level in savanna areas of Central Brazil and impacts of fire. Journal of Tropical Ecology, Cambridge, v. 22, n. 2, p. 191-201, 2006.

NERI, A. V.; MEIRA NETO, J. A. A.; SILVA, A. F. A. Woody community structure in a Cerrado sensu stricto area of the municipality of Senador Modestino Gonçalves, north of Minas Gerais State, Brazil. Revista Árvore, Viçosa, MG, v. 31, n. 1, p. 123-134, 2007.

RATTER, J. A.; DARGIE, T. C. D. An analysis of floristic composition of 26 Cerrado areas in Brazil. Edinburgh Journal of Botany, Edinburgh, v. 49, n. 2, p. 235-250, 1992.

SALLES, J. C.; SCHIAVINI, I. Structure and composition of the regeneration layer in an urban forest fragment: implications for the dynamics and conservation of the tree community. Acta Botanica Brasilica, São Paulo, v. 21, n. 1, p. 223-233, 2007.

SANTANA, O. A.; IMAÑA-ENCINAS, J. Modelo espacial de contaminação do solo e do lençol freático do aterro do Jockey Club para o parque nacional de Brasília. Brasília - DF. In: SIMPÓSIO BRASILEIRO DE CARTOGRAFIA GEOTÉCNICA E GEOAMBIENTAL, 5.; 2004; SÃO CARLOS, SP. Cartografia geotécnica e geoambiental: conhecimento do meio físico: base para a sutentabilidade). São Carlos: ABGE, 2004. p. 453-460.

SANTOS, H. G.; COELHO, M. R.; ANJOS, L. H. C.; JACOMINE, P. K. T.; OLIVEIRA, V. A.; LUMBRERAS, J. F.; OLIVEIRA, J. B.; CARVALHO, A. P.; FASOLO, P. J. Propostas de revisão e atualização do Sistema Brasileiro de Classificação de Solos. Rio de Janeiro: Embrapa Solos, 2003. 56 p.

SAPORETTI Jr., A. W.; MEIRA NETO, J. A. A.; ALMADO, R. Fitossociologia de sub-bosque de Cerrado em talhão de Eucalyptus grandis W. Hill ex Maiden no município de Bom Despacho-MG. 2003. Revista Árvore, Viçosa, MG, v. 27, n. 6, p. 905-910, 2003.

SHEPHERD, G. J. Manual do Fitopac. Campinas: UNICAMP, 1987. 93 p.

SILBERBAUER-GOTTSBERGER, I.; EITEN, G. Fitossociologia de um hectare de Cerrado. Brasil Florestal, Brasília, DF, v. 54, p. 55-70, 1983. 
SILVA, L. O.; COSTA, D. A.; ESPÍRITO SANTO FILHO, K.; FERREIRA, H. D.; BRANDÃO, D. Levantamento florístico e fitossociológico em duas áreas de Cerrado sensu stricto no Parque Estadual da Serra de Caldas Novas, Goiás. Acta Botanica Brasilica, São Paulo, v. 16, n. 1, p. 43-53, 2002.

SILVA JÚNIOR, M. C. Fitossociologia e estrutura diamétrica da mata de galeria do Taquara, na reserva ecológica do IBGE, DF. Revista Árvore, Viçosa, MG, v. 28, n. 3, p. 419-428, 2004.

TEIXEIRA, M. I. J. G.; ARAUJO, A. R. B.; VALERI, S. V. Floristic and phytosociology of area of Cerrado "scrictu sensu", in northeast region of São Paulo State, Brazil. Bragantia, Campinas, v. 63, n. 1, p. 1-11, 2004.

ZAR, J. H. Biostatistical analysis. 4th ed. New Jersey, USA: Prentice Hall, 1999. 123 p. 